UPRF 93-382

July 1993

\title{
Ward identities and Wilson renormalization group for QED"̈
}

\author{
M. Bonini, M. D’Attanasio and G. Marchesini \\ Dipartimento di Fisica, Università di Parma and \\ INFN, Gruppo Collegato di Parma, Italy
}

\begin{abstract}
We analyze a formulation of QED based on the Wilson renormalization group. Although the "effective Lagrangian" used at any given scale does not have simple gauge symmetry, we show that the resulting renormalized Green's functions correctly satisfies Ward identities to all orders in perturbation theory. The loop expansion is obtained by solving iteratively the Polchinski's renormalization group equation. We also give a new simple proof of perturbative renormalizability. The subtractions in the Feynman graphs and the corresponding counterterms are generated in the process of fixing the physical conditions.
\end{abstract}

\footnotetext{
${ }^{*}$ Research supported in part by MURST, Italy
} 


\section{Introduction}

In a gauge theory the presence of ultraviolet (UV) divergences could conflict with local gauge symmetry. There are two cases in which this conflict is avoided: dimensional regularization, which is applicable only in perturbation theory, and the Wilson formulation of lattice gauge theories [1] in which the Lagrangian is not local. However for chiral gauge theories even in these two approaches one is forced to introduce all possible counterterms, even those which break the local gauge symmetry [2, 3].

The most physical way to deal with UV divergences and to define the theory even beyond the perturbative expansion is given by the Wilson renormalization group equations [4]. In this method one starts with the Lagrangian at the UV scale $\Lambda_{0}$ which does not have simple gauge symmetry properties. Then one deduces the flow of the "effective Lagrangian" at a lower momentum scale $\Lambda$ by performing the path integrals over the fields with frequencies between $\Lambda$ and $\Lambda_{0}$. By integrating over all frequencies (i.e. by setting $\Lambda=0$ ) one obtains the physical "effective action" which should satisfy the Ward or Slavnov-Taylor identities. It is interesting to analyze in detail how these identities are violated along the renormalization group flow but are satisfied for the final result (i.e. $\Lambda=0$ and $\Lambda_{0} \rightarrow \infty$ ).

In this paper we study the Wilson renormalization group flow of QED and analyze how the Ward identities are fulfilled. We use the formulation given by Polchinski [5] (see also [6, 7] and for recent applications [8]-[12]). From this one deduces a linear differential equation in the scale $\Lambda$ for a functional $\Gamma[A, \psi, \bar{\psi} ; \Lambda]$ of the QED classical fields $A_{\mu}, \psi$ and $\bar{\psi}$ (for the case of a scalar theory see Refs. [10, 11]). The expansion coefficients of this functional are the "cutoff proper vertices" which are obtained by performing the path integrals over the fields with frequencies between $\Lambda$ and $\Lambda_{0}$, i.e. by cutting off the fields with frequencies outside this range. One has therefore that the physical effective action, given by performing the path integrals over all frequency range, are formally obtained by setting $\Lambda=0$ and taking the limit $\Lambda_{0} \rightarrow \infty$.

Given the evolution equation in $\Lambda$, the vertices of $\Gamma[A, \psi, \bar{\psi} ; \Lambda]$ are determined by supplementing appropriate boundary conditions. As we shall discuss in this paper this is the place in which the gauge symmetry properties must be implemented. The boundary conditions for the various vertices in $\Gamma[A, \psi, \bar{\psi} ; \Lambda]$ depend on their dimension in mass. Vertices with non negative mass dimension are called "relevant", while the others are called "irrelevant". Notice that they are irrelevant from the point of view of dimensional counting but they actually describe the interaction. As we shall see the relevant vertices reduce to seven couplings. One assumes the following boundary conditions:

1) at $\Lambda=\Lambda_{0}$ all "irrelevant" vertices vanish. This corresponds to assume that, at the UV scale, the vertices with negative mass dimension become proportional to inverse powers of $\Lambda_{0}$

2 ) at the physical value $\Lambda=0$ the "relevant" part of the effective action $\Gamma_{r e l}[A, \psi, \bar{\psi} ; \Lambda=0]$ 
is given by the classical action, e.g. in the Feynman gauge

$$
\begin{aligned}
& \Gamma_{r e l}\left[A_{\mu}, \psi, \bar{\psi} ; \Lambda=0\right]=\int_{p}\left\{\frac{1}{2} A_{\mu}(-p) p^{2} \delta_{\mu \nu} A_{\nu}(p)+i \bar{\psi}(-p)(\not p+m) \psi(p)\right\} \\
& +i e \int_{p} \int_{q} \bar{\psi}(-p) A(q) \psi(p-q), \quad \int_{p} \equiv \int \frac{d^{4} p}{(2 \pi)^{4}} .
\end{aligned}
$$

In this way one fixes the seven relevant couplings at $\Lambda=0$ to be the physical mass and charge, and to satisfy Ward identities.

The main result of this paper is the proof, in perturbation theory, that the functional $\Gamma[A, \psi, \bar{\psi} ; \Lambda]$ obtained from the renormalization group equation in $\Lambda$ and the above boundary conditions, satisfies Ward identities in the physical limit $\Lambda_{0} \rightarrow \infty$ and $\Lambda=0$.

The loop expansion for the vertices of $\Gamma[A, \psi, \bar{\psi} ; \Lambda]$ are deduced by solving iteratively the evolution equation in $\Lambda$. They are given by the usual Feynman diagrams in which all propagators have Euclidean momentum $p$ in the range $\Lambda^{2}<p^{2}<\Lambda_{0}^{2}$. The subtractions needed in order to take the limit $\Lambda_{0} \rightarrow \infty$ are automatically generated in the process of fixing the physical conditions in (11) for the "relevant" vertices. The fact that Ward identities are satisfied in the physical limit $\Lambda_{0} \rightarrow \infty$ and $\Lambda=0$ is deeply connected to the property of perturbative renormalizability, namely to the fact that the divergences in the non subtracted contributions of Feynman diagrams affect only the relevant vertices. Thus UV divergences are tamed by imposing the boundary conditions which generate the necessary subtractions. The perturbative proof of Ward identities is then obtained by showing that at $\Lambda=0$ the non subtracted vertices violate these identities for $\Lambda_{0} \rightarrow \infty$ only for "relevant" contributions which are automatically cancelled by imposing the physical conditions. For a previous analysis see for instance [8, 13].

The paper is organized as follows. First in Sect. 2 we deduce the evolution equation for the functional $\Gamma[A, \psi, \bar{\psi} ; \Lambda]$ and discuss in details the boundary conditions. In Sect. 3 we describe how the loop expansion is obtained from the iterative solution of the evolution equation for $\Gamma[A, \psi, \bar{\psi} ; \Lambda]$. We explicitly compute to one loop order all the vertices together with the axial anomaly diagram. To this order we show that the limit $\Lambda_{0} \rightarrow \infty$ can be taken, due to the proper subtractions, and that Ward identities are satisfied in the limits $\Lambda_{0} \rightarrow \infty$ and $\Lambda \rightarrow 0$. The proof to all perturbative order of these identities is presented

in Sect. 4. In Sect. 5 we formulate the proof of perturbative renormalizability. The last section contains some remarks and final comments.

\section{Renormalization group flow and effective action}

In order to compute the vertices of the effective action one needs a regularization procedure of the ultraviolet divergences. We regularize these divergences by assuming that in the path integral one integrates only the fields with frequencies smaller than a given UV cutoff $\Lambda_{0}$. This procedure is equivalent to assume that the free photon and electron propagators vanish for $p^{2}>\Lambda_{0}^{2}$. The physical theory is obtained by considering the limit $\Lambda_{0} \rightarrow \infty$. In order to study the Wilson renormalization group flow [4, 5], one introduces in the free propagators also an infrared (IR) cutoff $\Lambda$. The cutoff propagator for the electron and photon (in the 
Feynman gauge) are

$$
S_{\Lambda \Lambda_{0}}(p)=\frac{-i K_{\Lambda \Lambda_{0}}(p)}{\not p+m}, \quad \delta_{\mu \nu} D_{\Lambda \Lambda_{0}}(p)=\frac{\delta_{\mu \nu} K_{\Lambda \Lambda_{0}}(p)}{p^{2}},
$$

where $K_{\Lambda \Lambda_{0}}(p)=1$ in the region $\Lambda^{2} \lesssim p^{2} \lesssim \Lambda_{0}^{2}$ and rapidly vanishing outside. The corresponding free action is

$$
S_{0}^{\Lambda, \Lambda_{0}}=\int_{p}\left\{\frac{1}{2} A_{\mu}(-p) p^{2} \delta_{\mu \nu} A_{\nu}(p)+i \bar{\psi}(-p)(\not p+m) \psi(p)\right\} K_{\Lambda \Lambda_{0}}^{-1}(p) .
$$

The introduction of a cutoff in the propagators breaks the gauge invariance properties of the action. Therefore at the UV scale the interaction Lagrangian must contain all relevant couplings with non negative dimensions

$$
\begin{aligned}
S_{i n t}= & \int_{p} \frac{1}{2} A_{\mu}(-p)\left[\left(\sigma_{m_{A}}^{B}+p^{2} \sigma_{\alpha}^{B}\right) \delta_{\mu \nu}+p^{2} \sigma_{A}^{B} t_{\mu \nu}(p)\right] A_{\nu}(p)+\frac{\sigma_{4}^{B}}{8} \int d^{4} x\left(A^{2}(x)\right)^{2} \\
& +\int_{p} i \bar{\psi}(-p)\left[\sigma_{m_{\psi}}^{B}+\sigma_{\psi}^{B}(\not p+m)\right] \psi(p)+\int_{p} \int_{q} i \sigma_{e}^{B} \bar{\psi}(-p) A(q) \psi(p+q),
\end{aligned}
$$

where

$$
t_{\mu \nu}(p) \equiv \delta_{\mu \nu}-\frac{p_{\mu} p_{\nu}}{p^{2}}
$$

The photon and electron mass counterterms, $\sigma_{m_{A}}^{B}$ and $\sigma_{m_{\psi}}^{B}$, have positive dimensions, while the other parameters are dimensionless. $\sigma_{A}^{B}$ and $\sigma_{\psi}^{B}$ are related to the wave function renormalizations, $\sigma_{\alpha}^{B}$ to the gauge fixing parameter renormalization, $\sigma_{e}^{B}$ is related to the bare charge and $\sigma_{4}^{B}$ is the four photon interaction coupling. The complete Lagrangian $S^{\Lambda, \Lambda_{0}}=S_{0}^{\Lambda, \Lambda_{0}}+S_{\text {int }}$ violates the Ward identities, but the couplings $\sigma_{i}^{B}$ should be related in such a way that the (physical) effective action satisfies them.

In order to simplify the formulae we introduce the compact notation

$$
\begin{aligned}
& \Phi_{a}=\left(A_{\mu}, \psi_{\alpha}, \bar{\psi}_{\beta}\right), \quad J_{a}=\left(j_{\mu}, i \bar{\chi}_{\alpha},-i \chi_{\beta}\right), \\
& (J, \Phi) \equiv \int_{p} j_{\mu}(-p) A_{\mu}(p)+i \bar{\chi}(-p) \psi(p)+i \bar{\psi}(-p) \chi(p) .
\end{aligned}
$$

The free cutoff propagators are described by the matrix $D_{a b}$ defined by

$$
S_{0}^{\Lambda, \Lambda_{0}}=\int_{p} \frac{1}{2} \Phi_{a}(-p) D_{a b}^{-1}(p ; \Lambda) \Phi_{b}(p),
$$

with $S_{0}^{\Lambda, \Lambda_{0}}$ given by (2). The generating functional computed from the cutoff action $S^{\Lambda, \Lambda_{0}}$ will depend on $\Lambda_{0}$ and $\Lambda$. We have

$$
Z[J ; \Lambda]=\exp W[J ; \Lambda]=\int D\left[\Phi_{a}\right] \exp \left\{-S^{\Lambda, \Lambda_{0}}+(J, \Phi)\right\}
$$

where in $Z[J ; \Lambda]$ and $D_{a b}(p ; \Lambda)$ we have explicitly written only the cutoff $\Lambda$ since we will consider in any case the limit $\Lambda_{0} \rightarrow \infty$. The physical functional $Z[J]$ is obtained by taking the limits $\Lambda \rightarrow 0$ and $\Lambda_{0} \rightarrow \infty$. In these limits the "bare" parameters in (3) have to be

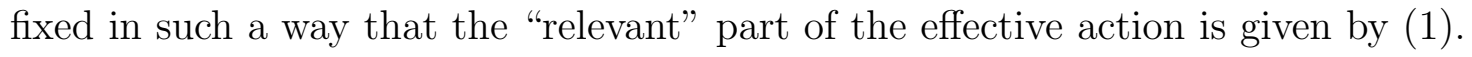




\subsection{Evolution equation}

Taking into account that all the $\Lambda$ dependence of $Z[J ; \Lambda]$ is coming from the cutoff in the propagators one easily derives [5] an evolution equation in $\Lambda$

$$
\Lambda \partial_{\Lambda} Z[J ; \Lambda]=-\frac{1}{2}(2 \pi)^{8} \int_{q} \Lambda \partial_{\Lambda} D_{b a}^{-1}(q ; \Lambda) \frac{\delta^{2} Z[J ; \Lambda]}{\delta J_{b}(q) \delta J_{a}(-q)} .
$$

This equation can be converted into an equation for the corresponding cutoff effective action $\Gamma[\Phi ; \Lambda]$ defined as the Legendre transformation of $W[J ; \Lambda]$

$$
\Gamma[\Phi ; \Lambda]=-W[J ; \Lambda]+W[0 ; \Lambda]+(J, \Phi), \quad \Phi_{a}(p)=(2 \pi)^{4} \frac{\delta W[J ; \Lambda]}{\delta J_{a}(-p)} .
$$

This can be done by isolating in $\Gamma[\Phi ; \Lambda]$ the contribution of the two point function

$$
(2 \pi)^{8} \frac{\delta^{2} \Gamma[\Phi]}{\delta \Phi_{a}(q) \delta \Phi_{b}\left(q^{\prime}\right)}=(2 \pi)^{4} \delta^{4}\left(q+q^{\prime}\right) \Delta_{b a}^{-1}(q ; \Lambda)+\Gamma_{b a}^{i n t}\left[q^{\prime}, q ; \Phi\right],
$$

where $\Delta_{a b}(q ; \Lambda)$ is the full propagator. In Appendix A we derive the evolution equation for the cutoff effective action and obtain

$$
\Lambda \partial_{\Lambda}\left\{\Gamma[\Phi ; \Lambda]-\frac{1}{2} \int_{q} \Phi_{a}(-q) D_{a b}^{-1}(q ; \Lambda) \Phi_{b}(q)\right\}=-\frac{1}{2} \int_{q} M_{b a}(q ; \Lambda) \bar{\Gamma}_{a b}[-q, q ; \Phi ; \Lambda],
$$

where

$$
M_{b a}=\Delta_{b c}(q ; \Lambda) \Lambda \partial_{\Lambda} D_{c d}^{-1}(q ; \Lambda) \Delta_{d a}(q ; \Lambda)
$$

and $\bar{\Gamma}_{a b}\left[q, q^{\prime} ; \Phi\right]$ is the auxiliary functional defined by the integral equation (see Fig. 1)

$$
\bar{\Gamma}_{a b}\left[q, q^{\prime} ; \Phi\right]=(-)^{\delta_{b}} \Gamma_{a b}^{i n t}\left[q, q^{\prime} ; \Phi\right]-\int_{q^{\prime \prime}} \bar{\Gamma}_{c b}\left[-q^{\prime \prime}, q^{\prime} ; \Phi\right] \Delta_{d c}\left(q^{\prime \prime} ; \Lambda\right) \Gamma_{a d}^{i n t}\left[q, q^{\prime \prime} ; \Phi\right],
$$

where $\delta_{b}$ is one if $b$ is a fermion index and zero otherwise. In terms of the proper vertices of $\Gamma[\Phi ; \Lambda]$ the evolution equations are $(n \geq 3)$

$$
\Lambda \partial_{\Lambda} \Gamma_{c_{1} \cdots c_{n}}\left(p_{1}, \cdots p_{n} ; \Lambda\right)=-\frac{1}{2} \int_{q} M_{b a}(q ; \Lambda) \bar{\Gamma}_{a b, c_{1} \cdots c_{n}}\left(-q, q ; p_{1}, \cdots p_{n} ; \Lambda\right),
$$

where $\bar{\Gamma}_{a b, c_{1} \cdots c_{n}}\left(q, q^{\prime} ; p_{1}, \cdots p_{n} ; \Lambda\right)$ are the vertices of the auxiliary functional $\bar{\Gamma}[\Phi ; \Lambda]$. For the two point function we write

$$
\Delta_{a b}^{-1}(q ; \Lambda)=D_{a b}^{-1}(q ; \Lambda)+\Pi_{a b}(q ; \Lambda)
$$

and one has

$$
\Lambda \partial_{\Lambda} \Pi_{c c^{\prime}}(p ; \Lambda)=-\frac{1}{2} \int_{q} M_{b a}(q ; \Lambda) \bar{\Gamma}_{a b, c c^{\prime}}(-q, q ;-p, p ; \Lambda) .
$$

The vertices of the auxiliary functional are obtained in terms of the proper vertices by expanding (8) and one finds

$$
\begin{aligned}
& \bar{\Gamma}_{a b, c_{1} \cdots c_{n}}\left(q, q^{\prime} ; p_{1}, \cdots p_{n} ; \Lambda\right)=\Gamma_{a b c_{1} \cdots c_{n}}\left(q, q^{\prime}, p_{1}, \cdots p_{n} ; \Lambda\right) \\
& -\sum^{\prime} \Gamma_{a c_{i_{1}} \cdots c_{i_{k}} c^{\prime}}\left(q, p_{i_{1}}, \cdots, p_{i_{k}}, Q ; \Lambda\right) \Delta_{c^{\prime} c^{\prime \prime}}(Q ; \Lambda) \bar{\Gamma}_{c^{\prime \prime} b, c_{i_{k+1}} \cdots c_{i_{n}}}\left(-Q, q^{\prime} ; p_{i_{k+1}}, \cdots p_{i_{n}} ; \Lambda\right)
\end{aligned}
$$

where $Q=q+p_{i_{1}}+\cdots p_{i_{k}}$, and $\sum^{\prime}$ is the sum over the combinations of photon and fermion indices $\left(i_{1} \cdots i_{n}\right)$ taking properly into account the symmetrization and anti-symmetrization. 


\subsection{Boundary conditions}

The evolution equation for $\Gamma[\Phi ; \Lambda]$ has to be supplemented by the appropriated boundary conditions for the relevant couplings at $\Lambda=0$ and for the "irrelevant" vertices at $\Lambda=\Lambda_{0}$. The relevant part of the effective action can be written in terms of the seven parameters as follows

$$
\begin{aligned}
& \Gamma_{r e l}[\Phi ; \Lambda]=\frac{1}{2} \int_{p} \Phi_{a}(-p) D_{a b}^{-1}(p ; \Lambda) \Phi_{b}(p)+\int_{p} i \bar{\psi}(-p)\left[\sigma_{m_{\psi}}(\Lambda)+\sigma_{\psi}(\Lambda)(p+m)\right] \psi(p) \\
& +\int_{p} \frac{1}{2} A_{\mu}(-p)\left[\left(\sigma_{m_{A}}(\Lambda)+p^{2} \sigma_{\alpha}(\Lambda)\right) \delta_{\mu \nu}+p^{2} \sigma_{A}(\Lambda) t_{\mu \nu}(p)\right] A_{\nu}(p) \\
& +\int_{p} \int_{q} i \sigma_{e}(\Lambda) \bar{\psi}(-p) A(q) \psi(p+q)+\frac{\sigma_{4}(\Lambda)}{8} \int d^{4} x\left(A^{2}(x)\right)^{2} .
\end{aligned}
$$

As described in the Introduction, the boundary conditions are:

1) at $\Lambda=0$

$$
\sigma_{e}(0)=e, \quad \sigma_{i}(0)=0, \quad \text { for } i=m_{A}, m_{\psi}, \alpha, A, \psi, 4 \text {; }
$$

2) at $\Lambda=\Lambda_{0}$

$$
\Gamma_{\text {irrel }}\left[\Phi ; \Lambda=\Lambda_{0}\right]=0,
$$

with $\Gamma=\Gamma_{\text {rel }}+\Gamma_{\text {irrel }}$. Ward identities are satisfied then at $\Lambda=0$ for the relevant part of the effective action. All couplings with negative dimension entering in $\Gamma_{\text {irrel }}[\Phi ; \Lambda]$ can be neglected for large $\Lambda$.

We analyse in detail these boundary conditions.

1) The photon propagator has the form

$$
\Delta_{\mu \nu}^{-1}(p ; \Lambda)=\delta_{\mu \nu} p^{2} K_{\Lambda \Lambda_{0}}^{-1}(p)+\Pi_{\mu \nu}(p ; \Lambda), \quad \Pi_{\mu \nu}(p ; \Lambda)=\delta_{\mu \nu} \Pi_{L}(p ; \Lambda)+t_{\mu \nu}(p) \Pi_{T}(p ; \Lambda)
$$

with the longitudinal and transverse components given by

$$
\Pi_{L}(p ; \Lambda)=\frac{p_{\mu} p_{\nu}}{p^{2}} \Pi_{\mu \nu}(p ; \Lambda), \quad \Pi_{T}(p ; \Lambda)=\frac{1}{3}\left(\delta_{\mu \nu}-4 \frac{p_{\mu} p_{\nu}}{p^{2}}\right) \Pi_{\mu \nu}(p ; \Lambda) .
$$

Using the three relevant couplings in (12) we have

$$
\begin{gathered}
\Pi_{L}(p ; \Lambda)=\sigma_{m_{A}}(\Lambda)+p^{2} \sigma_{\alpha}(\Lambda)+\Sigma_{L}(p ; \Lambda), \quad \Pi_{T}(p ; \Lambda)=p^{2} \sigma_{A}(\Lambda)+\Sigma_{T}(p ; \Lambda), \\
\Sigma_{T, L}(0 ; \Lambda)=0, \quad \frac{\partial \Sigma_{T, L}(p ; \Lambda)}{\partial p^{2}}=0 \quad \text { at } \quad p^{2}=\mu^{2},
\end{gathered}
$$

where $\mu$ is a subtraction point. From this definition we can factorize in the vertices $\Sigma_{T, L}$ a dimensional function of $p$, thus they are "irrelevant". The boundary conditions are then

$$
\sigma_{m_{A}}(0)=\sigma_{\alpha}(0)=\sigma_{A}(0)=0, \quad \Sigma_{T, L}\left(p ; \Lambda_{0}\right)=0 .
$$

2) The fermion propagator has the form

$$
\begin{gathered}
S^{-1}(p ; \Lambda)=i(p+m) K_{\Lambda \Lambda_{0}}^{-1}(p)+\Pi(p ; \Lambda), \quad \Pi(p ; \Lambda)=i \sigma_{m_{\psi}}(\Lambda)+i(p+m) \sigma_{\psi}(\Lambda)+\Sigma(p ; \Lambda), \\
\Sigma(p ; \Lambda)=0 \quad \text { at } \quad \not p=-m, \quad \frac{\partial}{\partial p_{\mu}} \Sigma(p ; \Lambda)=0 \quad \text { at } \quad p=0,
\end{gathered}
$$


where $\sigma_{m_{\psi}}, \sigma_{\psi}$ are two relevant couplings and the vertex $\Sigma(p ; \Lambda)$ is irrelevant. The boundary conditions are then

$$
\sigma_{m_{\psi}}(0)=\sigma_{\psi}(0)=0, \quad \Sigma\left(p ; \Lambda_{0}\right)=0 .
$$

3) For the electron-photon vertex we have one relevant coupling

$$
\Gamma_{\mu}\left(p, p^{\prime} ; \Lambda\right)=i \gamma_{\mu} \sigma_{e}(\Lambda)+\Sigma_{\mu}\left(p, p^{\prime} ; \Lambda\right), \quad \Sigma_{\mu}(0,0 ; \Lambda)=0
$$

and the boundary conditions are

$$
\sigma_{e}(0)=e, \quad \Sigma_{\mu}\left(p, p^{\prime} ; \Lambda_{0}\right)=0 .
$$

4) For the four photon interaction vertex one has

$$
\begin{gathered}
\Gamma_{\mu_{1} \cdots \mu_{4}}\left(p_{1} \cdots p_{4} ; \Lambda\right)=\sigma_{4}(\Lambda)\left(\delta_{\mu_{1} \mu_{2}} \delta_{\mu_{3} \mu_{4}}+\delta_{\mu_{1} \mu_{3}} \delta_{\mu_{2} \mu_{4}}+\delta_{\mu_{1} \mu_{4}} \delta_{\mu_{2} \mu_{3}}\right)+\Sigma_{\mu_{1} \cdots \mu_{4}}\left(p_{1} \cdots p_{4} ; \Lambda\right) \\
\Sigma_{\mu_{1} \cdots \mu_{4}}(0,0,0,0 ; \Lambda)=0
\end{gathered}
$$

and the boundary conditions are

$$
\sigma_{4}(0)=0, \quad \Sigma_{\mu_{1} \cdots \mu_{4}}\left(p_{1} \cdots p_{4} ; \Lambda_{0}\right)=0 .
$$

5) For all other vertex functions the dimensions are negative and one has

$$
\Gamma_{c_{1} \cdots c_{n}}\left(p_{1} \cdots p_{n} ; \Lambda_{0}\right)=0 .
$$

Finally we observe that the matrix $M_{a b}(q ; \lambda)$ in (7) for the fermion and photon case can be written in the following form

$$
\begin{aligned}
M_{\alpha \beta}(q ; \lambda)= & -\left[1+S_{\Lambda \Lambda_{0}}(q) \Pi(q ; \lambda)\right]^{-2} \frac{-i}{q+m} \lambda \partial_{\lambda} K_{\lambda \Lambda_{0}}(q), \\
M_{\mu \nu}(q ; \lambda)= & -\left\{\left[1+D_{\Lambda \Lambda_{0}}(q) \Pi_{L}(q ; \lambda)\right]^{-2} \frac{q_{\mu} q_{\nu}}{q^{2}}\right. \\
& \left.+\left[1+D_{\Lambda \Lambda_{0}}(q)\left(\Pi_{L}(q ; \lambda)+\Pi_{T}(q ; \lambda)\right)\right]^{-2} t_{\mu \nu}(q)\right\} \frac{1}{q^{2}} \lambda \partial_{\lambda} K_{\lambda \Lambda_{0}}(q),
\end{aligned}
$$

where we used the propagators decompositions in (15) and (18). Notice that $\lambda \partial_{\lambda} K_{\lambda \Lambda_{0}}(q)$ is different from zero only for $q^{2} \simeq \lambda^{2}$, and is then independent of $\Lambda_{0}$.

\section{Loop expansion}

The loop expansion is obtained by solving iteratively (9) and (10). Here we compute the first loop. The starting point of the iteration is the zero loop order in which, from (13), the only non vanishing coupling is

$$
\sigma_{e}^{(0)}(\Lambda)=e
$$

The zero loop auxiliary vertex corresponding to the electron emitting $n$ photons is given by Fig. $2 \mathrm{a}$

$$
\bar{\Gamma}_{\alpha \beta, \mu_{1} \cdots \mu_{n}}^{(0)}\left(q, q^{\prime} ; p_{1} \cdots p_{n} ; \Lambda\right)=i(-e)^{n}\left\{I_{\mu_{1} \cdots \mu_{n}}^{\left(\Lambda \Lambda_{0}\right)}\left(q ; p_{1}, \cdots p_{n}\right)\right\}_{\alpha \beta}+\text { permutations }
$$


where $q^{\prime}$ is given by momentum conservation,

$$
\begin{aligned}
I_{\mu_{1} \cdots \mu_{n}}^{\left(\Lambda \Lambda_{0}\right)}\left(q ; p_{1}, \cdots p_{n}\right) & \equiv I_{\mu_{1} \cdots \mu_{n}}\left(q ; p_{1}, \cdots p_{n}\right) K_{\Lambda \Lambda_{0}}\left(q+p_{1}, \cdots, q+p_{1}+\cdots p_{n-1}\right), \\
I_{\mu_{1} \cdots \mu_{n}}\left(q ; p_{1}, \cdots p_{n}\right) & =\gamma_{\mu_{1}} \frac{1}{\not 1+p_{1}+m} \gamma_{\mu_{2}} \cdots \frac{1}{\not 1+\not p_{1}+\cdots p_{n-1}+m} \gamma_{\mu_{n}}
\end{aligned}
$$

and we have introduced the general cutoff function

$$
K_{\Lambda \Lambda_{0}}\left(q_{1}, q_{2}, \cdots, q_{n}\right) \equiv K_{\Lambda \Lambda_{0}}\left(q_{1}\right) K_{\Lambda \Lambda_{0}}\left(q_{2}\right) \cdots K_{\Lambda \Lambda_{0}}\left(q_{n}\right) .
$$

The other zero loop auxiliary vertices are written in a similar way.

The one loop vertices are obtained by using in the right hand side of (6) the auxiliary vertices and propagators at zero loop. Once the boundary conditions are imposed, the one loop vertices are given in terms of subtracted integrands. Then all momentum integrations are UV convergent and we can take the limit $\Lambda_{0} \rightarrow \infty$. We show also that at $\Lambda=0$ and $\Lambda_{0} \rightarrow \infty$ the Ward identities are satisfied to this order.

\section{Electron propagator}

The one loop contribution for the electron propagator is obtained from the evolution equation (10) by using in the r.h.s. the zero loop contribution

$$
\Lambda \partial_{\Lambda} \Pi_{\alpha \beta}^{(1)}(p ; \Lambda)=-\frac{1}{2} \int_{q} M_{b a}^{(0)}(q ; \Lambda) \bar{\Gamma}_{a b, \alpha \beta}^{(0)}(-q, q ;-p, p ; \Lambda),
$$

where $M_{b a}^{(0)}=-\Lambda \partial_{\Lambda} D_{b a}$ and the auxiliary vertices $\bar{\Gamma}_{\alpha^{\prime} \beta^{\prime}, \alpha \beta}^{(0)}$ and $\bar{\Gamma}_{\mu \nu, \alpha \beta}^{(0)}$ are given in Fig. $2 \mathrm{~b}$. By noticing that there is a factor 2 for both cases coming from the permutations of the vertices in $\bar{\Gamma}$ (see Eq.(11)), the solution of Eq. (27) is

$$
\Pi^{\prime}(p ; \Lambda)=i e^{2} \int_{q} \frac{1}{q^{2}} \gamma_{\rho} \frac{1}{\not p+\not q+m} \gamma_{\rho} K_{\Lambda \Lambda_{0}}(q, q+p),
$$

apart from a term constant in $\Lambda$. This is fixed by imposing the boundary conditions (19) and we obtain the one loop contribution

$$
\Pi^{(1)}(p ; \Lambda)=\Pi^{\prime}(p ; \Lambda)-\Pi^{\prime}(\bar{p} ; 0)-\left.\left(p_{\mu}-\frac{m}{4} \gamma_{\mu}\right) \partial_{p_{\mu}^{\prime}} \Pi^{\prime}\left(p^{\prime} ; 0\right)\right|_{p^{\prime}=0}, \quad \not p=-m .
$$

Because of these subtractions, we can take the limit $\Lambda_{0} \rightarrow \infty$

$$
\begin{aligned}
\Pi^{(1)}(p ; \Lambda)= & i e^{2} \int_{q} \frac{1}{q^{2}}\left\{\gamma_{\rho} \frac{1}{p+\not q+m} \gamma_{\rho}-\gamma_{\rho} \frac{1}{\not p+\not q+m} \gamma_{\rho}+\left(p_{\mu}-\frac{m}{4} \gamma_{\mu}\right) \chi_{\mu}(0,0, q)\right\} \\
& +i e^{2} \int_{q} \frac{1}{q^{2}} \gamma_{\rho} \frac{1}{\not p+\not q+m} \gamma_{\rho}\left(K_{\Lambda \infty}(q, q+p)-1\right)
\end{aligned}
$$

where

$$
\chi_{\mu}\left(p, p^{\prime}, q\right) \equiv \gamma_{\rho} \frac{1}{\not q+\not p+m} \gamma_{\mu} \frac{1}{\not 1+\not p^{\prime}+m} \gamma_{\rho} .
$$

The last term, which is $\Lambda$-dependent, is convergent due to the finite range of integration coming from the IR cutoff. As we shall see this term is violating the Ward identities but is vanishing at the physical value $\Lambda=0$ since $K_{\Lambda \infty}(p) \rightarrow 1$ for $\Lambda \rightarrow 0$. 
By using a sharp momentum cutoff in (28), we find that for $\Lambda \rightarrow \infty$ the relevant couplings (i.e. the parameters in the bare Lagrangian) are given by

$$
\sigma_{m_{\psi}}^{(1)}(\Lambda)=\frac{3 e^{2}}{16 \pi^{2}} m \log \frac{\Lambda^{2}}{m^{2}}+\mathcal{O}(\infty), \quad \sigma_{\psi}^{(\infty)}(*)=\frac{\rceil^{\epsilon}}{\infty \mathbb{\pi}^{\epsilon}} \log \frac{*^{\epsilon}}{\mathbb{1}}+\mathcal{O}(\infty) .
$$

In the following we will compute the other bare parameters by using the same cutoff function.

\section{Electron vertex}

For this vertex one proceeds as before. The evolution equation (9) and the conditions (20) give

$$
\begin{gathered}
\Gamma_{\mu}^{(1)}\left(p, p^{\prime} ; \Lambda\right)=\Gamma_{\mu}^{\prime}\left(p, p^{\prime} ; \Lambda\right)-\Gamma_{\mu}^{\prime}(0,0 ; 0), \\
\Gamma_{\mu}^{\prime}\left(p, p^{\prime} ; \Lambda\right)=-i e^{3} \int_{q} \frac{1}{q^{2}} \chi_{\mu}\left(p, p^{\prime}, q\right) K_{\Lambda \Lambda_{0}}\left(q, q+p, q+p^{\prime}\right) .
\end{gathered}
$$

By taking the limit $\Lambda_{0} \rightarrow \infty$ we find

$$
\begin{aligned}
\Gamma_{\mu}^{(1)}\left(p, p^{\prime} ; \Lambda\right)= & -i e^{3} \int_{q} \frac{1}{q^{2}}\left\{\chi_{\mu}\left(p, p^{\prime}, q\right)-\chi_{\mu}(0,0, q)\right\} \\
& -i e^{3} \int_{q} \frac{1}{q^{2}} \chi_{\mu}\left(p, p^{\prime}, q\right)\left\{K_{\Lambda \infty}\left(q, q+p, q+p^{\prime}\right)-1\right\} .
\end{aligned}
$$

As before the last term is violating Ward identities but is vanishing for $\Lambda \rightarrow 0$.

For large values of $\Lambda$ we find

$$
\sigma_{e}^{(1)}(\Lambda)=\frac{e^{3}}{16 \pi^{2}} \log \frac{\Lambda^{2}}{m^{2}}+\mathcal{O}(\infty)
$$

Notice that at the leading order in $\Lambda$ we have $\sigma_{e}^{(1)}=\sigma_{\psi}^{(1)}$, however this relation is violated by the finite parts. As anticipated the action at the UV scale is not gauge invariant.

\section{Ward identity for electron propagator and vertex}

The fact that at $\Lambda=0$ the electron propagator and electron-photon vertex satisfy the Ward identity can be shown by using the identity

$$
\left(p^{\prime}-p\right)_{\mu} \chi_{\mu}\left(p, p^{\prime}, q\right)=\gamma_{\rho}\left(\frac{1}{\not q+\not p+m}-\frac{1}{\not q+\not p^{\prime \prime}+m}\right) \gamma_{\rho}
$$

From this we obtain

$$
\begin{aligned}
& \left(p^{\prime}-p\right)_{\mu} \Gamma_{\mu}^{(1)}\left(p, p^{\prime} ; \Lambda\right)-e\left(\Pi^{(1)}\left(p^{\prime} ; \Lambda\right)-\Pi^{(1)}(p ; \Lambda)\right) \\
& =-i e^{3} \int_{q} \frac{1}{q^{2}} \gamma_{\rho} \frac{K_{\Lambda \infty}(q, p+q)}{\not 1+\not p+m} \gamma_{\rho}\left[K_{\Lambda \infty}\left(p^{\prime}+q\right)-1\right]-\left\{p \rightarrow p^{\prime}\right\} .
\end{aligned}
$$

For $\Lambda=0$ the r.h.s. vanishes thus the Ward identity is satisfied. Notice that for $\Lambda \neq 0$ and finite the violation is not a polynomial in $p$ and $p^{\prime}$ and therefore receives contribution both from the relevant and the irrelevant couplings. 


\section{Photon propagator and Ward identity}

For the longitudinal and the transverse part of the photon propagator at one loop the evolution equation (9) and the boundary conditions (17) give

$$
\begin{aligned}
& \Pi_{L, T}^{(1)}(p ; \Lambda)=\Pi_{L, T}^{\prime}(p ; \Lambda)-\Pi_{L, T}^{\prime}(0 ; 0)-p^{2} \frac{\partial}{\partial \bar{p}^{2}} \Pi_{L, T}^{\prime}(\bar{p} ; 0), \quad \bar{p}^{2}=\mu^{2} \\
& \Pi_{L, T}^{\prime}(p ; \Lambda)=e^{2} \int_{q} I_{L, T}(q ; p) K_{\Lambda \Lambda_{0}}(q, q+p)
\end{aligned}
$$

with

$$
\begin{aligned}
& I_{L}(q ; p)=\operatorname{Tr}\left(\frac{1}{q+m} \frac{p_{\mu} p_{\nu}}{p^{2}} I_{\mu \nu}(q ; p,-p)\right) \\
& I_{T}(q ; p)=\frac{1}{3}\left(\delta_{\mu \nu}-4 \frac{p_{\mu} p_{\nu}}{p^{2}}\right) \operatorname{Tr}\left(\frac{1}{\not+m} I_{\mu \nu}(q ; p,-p)\right),
\end{aligned}
$$

where $I_{\mu \nu}$ is defined in (26) and we have $\Pi_{T}^{\prime}(0 ; 0)=0$. By taking the limit $\Lambda_{0} \rightarrow \infty$ we find

$$
\Pi_{L, T}^{(1)}(p ; \Lambda)=e^{2} \int_{q} I_{L, T}^{s}(q ; p)+e^{2} \int_{q} I_{L, T}(q ; p)\left(K_{\Lambda \infty}(q, q+p)-1\right)
$$

where $I_{L, T}^{s}$ are subtracted integrands

$$
I_{L, T}^{s}(q ; p)=I_{L, T}(q ; p)-I_{L, T}(q ; 0)-p^{2} \frac{\partial}{\partial \bar{p}^{2}} I_{L, T}(q ; \bar{p})
$$

For large $\Lambda$ the relevant couplings are given by

$$
\sigma_{m_{A}}^{(1)}(\Lambda)=\frac{e^{2}}{8 \pi^{2}} \Lambda^{2}+\mathcal{O}(\infty), \quad \sigma_{\alpha}^{(\infty)}(*)=\mathcal{O}\left(\frac{\infty}{*^{\epsilon}}\right), \quad \sigma_{\mathcal{A}}^{(\infty)}(*)=-\frac{\rceil^{\epsilon}}{\infty \in \pi^{\epsilon}} \log \frac{*^{\epsilon}}{\mathbb{1}}+\mathcal{O}(\infty) .
$$

We now show that to one loop order for $\Lambda=0$ the physical propagator is purely transverse $\left(\Pi_{L}^{(1)}(p ; 0)=0\right)$. To show this, in Eq. (33) one writes

$$
I_{L}(q ; p)=\frac{1}{p^{2}} \operatorname{Tr}\left(\not p \frac{1}{\not 1+m}-\not p \frac{1}{\not q+\not p+m}\right),
$$

changes integration variable in the second term and obtains

$$
\Pi_{L}^{\prime}(p ; 0)=e^{2} \frac{1}{p^{2}} \int_{q} \operatorname{Tr}\left(\not p \frac{1}{\not q+m}\right)\left(K_{0 \Lambda_{0}}(q, q+p)-K_{0 \Lambda_{0}}(q, q-p)\right) .
$$

Due to the difference of the two cutoff functions we have that $q^{2}$ is forced in the region $q^{2} \sim \Lambda_{0}$. By taking for instance an exponential UV cutoff one has

$$
K_{0 \Lambda_{0}}(q+p)-K_{0 \Lambda_{0}}(q-p)=-4 \frac{p \cdot q}{\Lambda_{0}^{2}}\left\{1-\frac{p^{2}}{\Lambda_{0}^{2}}+\frac{2}{3} \frac{(p \cdot q)^{2}}{\Lambda_{0}^{4}}+\cdots\right\} e^{-q^{2} / \Lambda_{0}^{2}}
$$

and obtains

$$
\Pi_{L}^{\prime}(p ; 0)=-\frac{e^{2}}{16 \pi^{2}}\left(\Lambda_{0}^{2}-2 m^{2}\right)+\frac{e^{2}}{24 \pi^{2}} p^{2}+\mathcal{O}\left(\frac{m^{2}, p^{2}}{\Lambda_{0}^{2}}\right)
$$


In this calculation the effect of the non invariant regularization is clear. The divergent integral with the cutoff functions gives a surface term which destroys the transversality of the propagator. However the longitudinal contributions are of relevant type, thus they are cancelled by imposing the boundary conditions. One finds

$$
\Pi_{L}^{(1)}(p ; 0)=\Pi_{L}^{\prime}(p ; 0)-\Pi_{L}^{\prime}(0 ; 0)-\left.p^{2} \partial_{p^{\prime 2}} \Pi_{L}^{\prime}\left(p^{\prime} ; 0\right)\right|_{p^{\prime 2}=\mu^{2}}=\mathcal{O}\left(\frac{m^{2}, p^{2}}{\Lambda_{0}^{2}}\right) \rightarrow 0 .
$$

For $\Lambda \neq 0$ the longitudinal part of the photon propagator is different from zero both in the relevant $\left(\sigma_{m_{A}}(\Lambda)\right.$ and $\left.\sigma_{\alpha}(\Lambda)\right)$ and irrelevant $\left(\Sigma_{L}(p ; \Lambda)\right)$ parts.

\section{Multi-photon vertices}

The evolution equation and the boundary conditions (22) and (23) give

$$
\Gamma_{\mu_{1} \cdots \mu_{n}}^{(1)}\left(p_{1}, \cdots p_{n} ; \Lambda\right)=\Gamma_{\mu_{1} \cdots \mu_{n}}^{\prime}\left(p_{1}, \cdots p_{n} ; \Lambda\right)-\Gamma_{\mu_{1} \cdots \mu_{n}}^{\prime}(0, \cdots 0 ; 0) \delta_{n, 4},
$$

where the subtraction is required only for the four photon vertex. The non subtracted vertices are

$$
\Gamma_{\mu_{1} \cdots \mu_{n}}^{\prime}\left(p_{1}, \cdots p_{n} ; \Lambda\right)=(-e)^{n} \int_{q} \operatorname{Tr}\left\{\frac{K_{\Lambda \Lambda_{0}}(q)}{\not q+m} I_{\mu_{1} \cdots \mu_{n}}^{\left(\Lambda \Lambda_{0}\right)}\left(q ; p_{1}, \cdots p_{n}\right)\right\}+\text { permutations }
$$

where $I_{\mu_{1} \cdots \mu_{n}}^{\left(\Lambda \Lambda_{0}\right)}$ is defined in (26). For $n>4$ this integral is convergent and we can remove the cutoff $\Lambda_{0}$. In the case $n=4$ one needs to consider the subtraction

$$
\begin{aligned}
& \Gamma_{\mu_{1} \cdots \mu_{4}}^{(1)}\left(p_{1}, \cdots p_{4} ; \Lambda\right)=\Gamma_{\mu_{1} \cdots \mu_{4}}^{\prime}\left(p_{1}, \cdots p_{4} ; \Lambda\right)-\Gamma_{\mu_{1} \cdots \mu_{4}}^{\prime}(0, \cdots 0 ; 0) \\
& =e^{4} \int_{q} \operatorname{Tr}\left\{\frac{1}{\not 1+m}\left(I_{\mu_{1} \cdots \mu_{4}}\left(q ; p_{1}, \cdots p_{4}\right)-I_{\mu_{1} \cdots \mu_{4}}(q ; 0, \cdots 0)\right)\right\} \\
& +e^{4} \int_{q} \operatorname{Tr}\left\{\frac{1}{\not q+m} I_{\mu_{1} \cdots \mu_{4}}\left(q ; p_{1}, \cdots p_{4}\right)\right\}\left[K_{\Lambda \infty}\left(q, q+p_{1} \cdots\right)-1\right]+\text { permutations } .
\end{aligned}
$$

The various terms in the non-subtracted vertex $\Gamma_{\mu_{1} \cdots \mu_{4}}^{\prime}$ are logarithmically divergent when removing the cutoff. However, by summing over the permutations, these divergent terms cancel, giving a finite result. In dimensional regularization, at vanishing momenta the vertex is zero. In the present regularization one finds instead a finite result for $\Lambda_{0} \rightarrow \infty$. At $\Lambda=0$ we have

$$
\Gamma_{\mu_{1} \cdots \mu_{4}}^{\prime}(0,0,0,0 ; 0)=\frac{e^{4}}{12 \pi^{2}}\left(\delta_{\mu_{1} \mu_{2}} \delta_{\mu_{3} \mu_{4}}+\delta_{\mu_{1} \mu_{3}} \delta_{\mu_{2} \mu_{4}}+\delta_{\mu_{1} \mu_{4}} \delta_{\mu_{2} \mu_{3}}\right)
$$

The non vanishing of this finite term implies that the subtraction required by the constraint (22) is necessary even if its contribution is finite. With this subtraction we have $\Gamma_{\mu_{1} \cdots \mu_{4}}(0, \cdots 0 ; 0)=0$ which is essential in order to satisfy the Ward identity. For large $\Lambda$ the relevant coupling is

$$
\sigma_{4}^{(1)}(\Lambda)=-\frac{e^{4}}{12 \pi^{2}}+\mathcal{O}\left(\frac{1}{\Lambda^{2}}\right)
$$

6. Ward identities for the multi-photon vertices 
The basic identity used to prove the Ward identities is

$$
p_{1 \mu_{1}} \frac{1}{\not q+m} I_{\mu_{1} \cdots \mu_{n}}\left(q ; p_{1}, \cdots p_{n}\right)=\left(\frac{1}{\not q+m}-\frac{1}{\not q+\not p_{1}+m}\right) I_{\mu_{2} \cdots \mu_{n}}\left(q+p_{1} ; p_{2}, \cdots p_{n}\right) .
$$

By using (41) in (37) and changing integration variable we can write

$$
\begin{aligned}
& p_{1 \mu_{1}} \Gamma_{\mu_{1} \cdots \mu_{n}}^{\prime}\left(p_{1}, \cdots p_{n} ; \Lambda\right)=(-e)^{n} \int_{q}\left[K_{\Lambda \Lambda_{0}}\left(q+p_{1}\right)-K_{\Lambda \Lambda_{0}}\left(q+p_{2}\right)\right] \\
& \times \operatorname{Tr}\left\{\frac{K_{\Lambda \Lambda_{0}}(q)}{\not 1+m} I_{\mu_{2} \mu_{3} \cdots \mu_{n}}^{\left(\Lambda \Lambda_{0}\right)}\left(q+p_{1} ; p_{2}, p_{3} \cdots p_{n}\right)\right\}+\cdots
\end{aligned}
$$

where the dots correspond to the sum over the permutations of $(2, \ldots n)$. Consider first the case $n>4$ which needs not subtractions. By expanding for $\Lambda=0$ the differences of cutoff functions, similarly to (36), we obtain the following behaviour for large $\Lambda_{0}$

$$
p_{1 \mu_{1}} \Gamma_{\mu_{1} \cdots \mu_{n}}^{(1)}\left(p_{1}, \cdots p_{n} ; 0\right)=c\left(p_{1 \mu_{2}} \delta_{\mu_{3} \mu_{4}} \cdots \delta_{\mu_{n-1} \mu_{n}}+\text { symm. }\right)\left(\frac{1}{\Lambda_{0}}\right)^{n-4}+\mathcal{O}\left(p_{1} \frac{P^{2}}{\Lambda_{0}^{n-2}}\right) \rightarrow 0,
$$

where $P$ is a combination of external momenta and $c$ a numerical constant. Notice that (43) implies

$$
\Gamma_{\mu_{1} \cdots \mu_{n}}^{(1)}(0, \cdots 0 ; 0)=0 .
$$

In the case $n=4$ one obtains a finite result

$$
p_{1 \mu_{1}} \Gamma_{\mu_{1} \cdots \mu_{4}}^{\prime}\left(p_{1}, \cdots p_{4} ; 0\right)=\frac{e^{4}}{12 \pi^{2}}\left(p_{1 \mu_{2}} \delta_{\mu_{3} \mu_{4}}+p_{1 \mu_{3}} \delta_{\mu_{2} \mu_{4}}+p_{1 \mu_{4}} \delta_{\mu_{3} \mu_{2}}\right)+\mathcal{O}\left(p_{1} \frac{P^{2}}{\Lambda_{0}^{2}}\right),
$$

where the constant term comes from the first term in the $1 / \Lambda_{0}$ expansion of the differences of cutoffs. Combining this with the result (39) one finds for $\Lambda=0$ and $\Lambda_{0} \rightarrow \infty$ that the Ward identity is satisfied to this order

$$
p_{1 \mu_{1}} \Gamma_{\mu_{1} \cdots \mu_{4}}^{(1)}\left(p_{1}, \cdots p_{4} ; 0\right)=\mathcal{O}\left(p_{1} \frac{P^{2}}{\Lambda_{0}^{2}}\right) \rightarrow 0
$$

\section{Axial anomaly}

Finally we consider the axial Ward identity for the VVA (axial) $T_{\mu \nu \rho}$ and the VVP (pseudoscalar) $T_{\nu \rho}$ vertices obtained by insertion of the axial $i \bar{\psi} \gamma_{\mu} \gamma_{5} \psi$ and pseudoscalar current $i \bar{\psi} \gamma_{5} \psi$ respectively

$$
\begin{aligned}
p_{\nu} T_{\mu \nu \rho}\left(p, p^{\prime}\right) & =0, \quad p_{\rho}^{\prime} T_{\mu \nu \rho}\left(p, p^{\prime}\right)=0, \\
\left(p+p^{\prime}\right)_{\mu} T_{\mu \nu \rho}\left(p, p^{\prime}\right) & =-2 m T_{\nu \rho}\left(p, p^{\prime}\right)-\frac{1}{2 \pi^{2}} \epsilon_{\alpha \nu \rho \beta} p_{\alpha} p_{\beta}^{\prime} .
\end{aligned}
$$

As well known at one loop the axial Ward identity develops an anomalous term.

Here we recover this result in our scheme. The generating functional for these vertices are obtained by adding the corresponding source terms in (4). Since these sources do not couple, no Legendre transformation is needed and the evolution equation for $T_{\mu \nu \rho}$ and $T_{\nu \rho}$ has the same structure of (9). The boundary conditions are fixed according to the same 
arguments of relevance previously discussed. The Lorentz covariance and symmetrization with respect to the two photons give the following decompositions

$$
\begin{aligned}
& T_{\mu \nu \rho}\left(p, p^{\prime} ; \Lambda\right)=\epsilon_{\mu \nu \rho \sigma}\left(p-p^{\prime}\right)_{\sigma} A\left(p, p^{\prime} ; \Lambda\right)+\epsilon_{\mu \nu \alpha \beta} p_{\alpha} p_{\beta}^{\prime}\left(p+p^{\prime}\right)_{\rho} \Sigma_{1}\left(p, p^{\prime} ; \Lambda\right) \\
& +\epsilon_{\mu \alpha \rho \beta} p_{\alpha} p_{\beta}^{\prime}\left(p+p^{\prime}\right)_{\nu} \Sigma_{1}\left(p^{\prime}, p ; \Lambda\right)+\epsilon_{\alpha \nu \rho \beta} p_{\alpha} p_{\beta}^{\prime}\left(p+p^{\prime}\right)_{\mu} \Sigma_{2}\left(p, p^{\prime} ; \Lambda\right)
\end{aligned}
$$

and

$$
T_{\mu \nu}\left(p, p^{\prime} ; \Lambda\right)=\epsilon_{\mu \nu \alpha \beta} p_{\alpha} p_{\beta}^{\prime} \Sigma_{3}\left(p, p^{\prime} ; \Lambda\right) .
$$

From dimensional counting we have that only the function $A$ has non-negative dimension and we can write

$$
A\left(p, p^{\prime} ; \Lambda\right)=\sigma_{5}(\Lambda)+\Sigma_{5}\left(p, p^{\prime} ; \Lambda\right),
$$

where $\sigma_{5}(\Lambda)$ is a relevant dimensionless constant while $\Sigma_{5}$ is irrelevant since $\Sigma_{5}(0,0 ; \Lambda)=0$. The boundary conditions are

$$
\sigma_{5}(0)=0, \quad \Sigma_{i}\left(p, p^{\prime} ; \Lambda_{0}\right)=0, \quad i=1,2,3,5 .
$$

The last condition is fixed by requiring that the relevant part of effective action satisfy the vector identity (45a). Imposing these boundary conditions we find, at one loop level

$$
T_{\mu \nu \rho}^{(1)}\left(p, p^{\prime} ; \Lambda\right)=T_{\mu \nu \rho}^{\prime}\left(p, p^{\prime} ; \Lambda\right)-\epsilon_{\mu \nu \rho \sigma}\left(p-p^{\prime}\right)_{\sigma} A^{\prime}(0,0 ; 0),
$$

where $T_{\mu \nu \rho}^{\prime}$ is the one loop graph

$$
\begin{gathered}
T_{\mu \nu \rho}^{\prime}\left(p, p^{\prime} ; \Lambda\right)=\int_{q} \operatorname{Tr} \frac{K_{\Lambda \Lambda_{0}}(q)}{\not 1+m} \gamma_{\mu} \gamma_{5} \frac{K_{\Lambda \Lambda_{0}}\left(q-p-p^{\prime}\right)}{\not 1-\not p-\not p^{\prime}+m} \gamma_{\rho} \frac{K_{\Lambda \Lambda_{0}}(q-p)}{\not q-\not p+m} \gamma_{\nu} \\
+(p, \nu) \rightarrow\left(p^{\prime}, \rho\right),
\end{gathered}
$$

and

$$
\epsilon_{\mu \nu \rho \sigma} A^{\prime}(0,0 ; \Lambda)=\left.\frac{\partial}{\partial p_{\sigma}} T_{\mu \nu \rho}^{\prime}\left(p, p^{\prime} ; \Lambda\right)\right|_{p=p^{\prime}=0} .
$$

The calculation of the Ward identities follows the same steps as the previous ones, in particular we must take into account the surface terms coming from the difference of cutoff functions. We find at $\Lambda=0$

$$
\begin{aligned}
p_{\nu} T_{\mu \nu \rho}^{\prime}\left(p, p^{\prime} ; 0\right) & =\frac{1}{6 \pi^{2}} \epsilon_{\mu \alpha \rho \beta} p_{\alpha} p_{\beta}^{\prime}+\mathcal{O}\left(\frac{\infty}{*_{,}^{\epsilon}}\right), \\
p_{\rho}^{\prime} T_{\mu \nu \rho}^{\prime}\left(p, p^{\prime} ; 0\right) & =\frac{1}{6 \pi^{2}} \epsilon_{\mu \nu \alpha \beta} p_{\alpha} p_{\beta}^{\prime}+\mathcal{O}\left(\frac{\infty}{*_{,}^{\epsilon}}\right), \\
\left(p+p^{\prime}\right)_{\mu} T_{\mu \nu \rho}^{\prime}\left(p, p^{\prime} ; 0\right) & =-\frac{1}{6 \pi^{2}} \epsilon_{\alpha \nu \rho \beta} p_{\alpha} p_{\beta}^{\prime}-2 m T_{\nu \rho}^{(1)}\left(p, p^{\prime} ; 0\right)+\mathcal{O}\left(\frac{\infty}{*_{,}^{\epsilon}}\right),
\end{aligned}
$$

where $T_{\nu \rho}^{(1)}$ is the one loop VVP vertex given by the r.h.s. of (49) with $\gamma_{\mu} \gamma_{5} \rightarrow \gamma_{5}$ and no subtraction is needed. Using (50a) and (50b) and a Lorentz decomposition of $T_{\mu \nu \rho}^{\prime}$ analogous to (46), we have

$$
A^{\prime}(0,0 ; 0)=-\frac{1}{6 \pi^{2}}+\mathcal{O}\left(\frac{1}{\Lambda_{0}^{2}}\right)
$$

In the limit $\Lambda_{0} \rightarrow \infty$, the Ward identities (45) are obtained from equation (48), after the insertion of (50) and (51).

In conclusion we have assumed the vector Ward identities in (45a) only for the relevant part of the VVA vertex. We have then proved that the two Ward identities in (45a)-45b) are satisfied for the physical VVA and VVP vertex. In particular the axial anomaly is generated by the surface contribution associated to a linear divergent graph. 


\section{$4 \quad$ Ward identities}

In this section we prove that the Ward identities are satisfied in perturbation theory when we take the physical limit. Therefore in this section we suppress the index $\Lambda$ which is set at $\Lambda=0$. We use the standard technique based on Feynman diagrams. The important point is the analysis of the role of the cutoff and the UV limit $\Lambda_{0} \rightarrow \infty$. As we shall see the non subtracted vertices violate the Ward identities by surfaces terms coming, as in the one loop case, from the difference of cutoff functions (36). We show that the violating terms are of relevant type thus they are cancelled by the subtractions coming by imposing the physical boundary conditions (13). The presence of the difference of cutoff functions (36) in non subtracted vertices forces the integration momenta to be of the order of the UV cutoff $\Lambda_{0}$. The evaluation of the integral is then obtained by using the Weinberg theorem [14 for the asymptotic behaviour at large momenta of the vertices in the integrands. We recall that this behaviour is given by power counting arguments, apart from logarithmic corrections, which are inessential for our perturbative analysis.

We consider first the fermion vertex identity and then the photon transversality.

\section{Fermion vertex}

Consider the general graphs Fig. 3a for the non subtracted fermion propagator which gives the contribution

$$
\Sigma^{\prime \mathcal{G}}(p)=i(-e)^{n} \int_{q_{1}} \cdots \int_{q_{n}}(2 \pi)^{4} \delta^{4}\left(\sum_{1}^{n} q_{i}\right) G_{\mu_{1} \cdots \mu_{n}}\left(q_{1} \cdots q_{n}\right) I_{\mu_{1} \cdots \mu_{n}}^{\left(0 \Lambda_{0}\right)}\left(p ; q_{1}, q_{2} \cdots q_{n}\right),
$$

where the function $G_{\mu_{1} \cdots \mu_{n}}\left(q_{1} \cdots q_{n}\right)$ corresponds to the sum of connected and disconnected Feynman graphs in the upper part of Fig. 3a. This function includes the photon propagators.

The corresponding graphs for the vertex function are obtained by inserting in Fig. 3a a photon of momentum $k=p^{\prime}-p$ with polarization $\mu$ in all possible ways. We obtain the two contributions of Fig. 4. From the first one in which the photon is inserted in the fermion line, we find

$$
\begin{aligned}
& k_{\mu} \Sigma_{\mu}^{\mathcal{G}}\left(p, p^{\prime}\right)=i(-e)^{n+1} \int_{q_{1}} \cdots \int_{q_{n}}(2 \pi)^{4} \delta^{4}\left(\sum_{1}^{n} q_{i}\right) G_{\mu_{1} \cdots \mu_{n}}\left(q_{1} \cdots q_{n}\right) \\
& \times k_{\mu} \sum_{\ell=1}^{n-1} I_{\mu_{1} \cdots \mu_{\ell} \mu \cdots \mu_{n}}^{\left(0 \Lambda_{0}\right)}\left(p ; q_{1}, \cdots q_{\ell}, k, \cdots q_{n}\right) .
\end{aligned}
$$

As we shall see later the contribution of the second type graphs vanish.

The contributions in (52) and (53) are not subtracted so that we should take $\Lambda_{0}$ fixed. The limit $\Lambda_{0} \rightarrow \infty$ can be considered only for the corresponding subtracted vertices

$$
\begin{aligned}
& \Sigma^{\mathcal{G}}(p)=\Sigma^{\prime \mathcal{G}}(p)-\Sigma^{\prime \mathcal{G}}(\bar{p})-\left.\left(p_{\mu}-\frac{m}{4} \gamma_{\mu}\right) \partial_{p_{\mu}^{\prime}} \Sigma^{\mathcal{G}}\left(p^{\prime}\right)\right|_{p^{\prime}=0}, \quad \not p=-m \\
& k_{\mu} \Sigma_{\mu}^{\mathcal{G}}\left(p, p^{\prime}\right)=k_{\mu}\left(\Sigma_{\mu}^{\mathcal{G}}\left(p, p^{\prime}\right)-\Sigma_{\mu}^{\mathcal{G}}(0,0)\right)
\end{aligned}
$$


obtained by imposing the boundary conditions (19) and (20). Subtraction of subdivergences are considered later. By using (52)-(54) the Ward identity violation

$$
\mathcal{W}^{\mathcal{G}}\left(p, p^{\prime} ; \Lambda_{0}\right)=k_{\mu} \Sigma_{\mu}^{\mathcal{G}}\left(p, p^{\prime}\right)-e\left(\Sigma^{\mathcal{G}}\left(p^{\prime}\right)-\Sigma^{\mathcal{G}}(p)\right)
$$

becomes

$$
\mathcal{W}^{\mathcal{G}}\left(p, p^{\prime} ; \Lambda_{0}\right)=i(-e)^{n+1} \int_{q_{1}} \cdots \int_{q_{n}}(2 \pi)^{4} \delta^{4}\left(\sum_{1}^{n} q_{i}\right) G_{\mu_{1} \cdots \mu_{n}}\left(q_{1} \cdots q_{n}\right) R_{\mu_{1} \cdots \mu_{n}}^{\left(\Lambda_{0}\right)}\left(p, p^{\prime}, q_{1} \cdots q_{n}\right)
$$

where

$$
\begin{aligned}
& R_{\mu_{1} \cdots \mu_{n}}^{\left(\Lambda_{0}\right)}\left(p, p^{\prime}, q_{1} \cdots q_{n}\right) \\
& =\sum_{\ell=1}^{n-1} k_{\mu}\left\{I_{\mu_{1} \cdots \mu_{\ell} \mu \cdots \mu_{n}}^{\left(0 \Lambda_{0}\right)}\left(p ; q_{1}, \cdots q_{\ell}, k, \cdots q_{n}\right)-I_{\mu_{1} \cdots \mu_{\ell} \mu \cdots \mu_{n}}^{\left(0 \Lambda_{0}\right)}\left(0 ; q_{1}, \cdots q_{\ell}, 0, \cdots q_{n}\right)\right\} \\
& -\left(I_{\mu_{1} \cdots \mu_{n}}^{\left(0 \Lambda_{0}\right)}\left(p ; q_{1}, \cdots q_{n}\right)-I_{\mu_{1} \cdots \mu_{n}}^{\left(0 \Lambda_{0}\right)}\left(p^{\prime} ; q_{1}, \cdots q_{n}\right)\right)-\left.k_{\mu} \partial_{p_{\mu}^{\prime \prime}} I_{\mu_{1} \cdots \mu_{n}}^{\left(0 \Lambda_{0}\right)}\left(p^{\prime \prime} ; q_{1}, \cdots q_{n}\right)\right|_{p^{\prime \prime}=0} .
\end{aligned}
$$

Without the cutoff one has the identity

$$
\begin{aligned}
& \sum_{\ell=1}^{n-1} k_{\mu}\left\{I_{\mu_{1} \cdots \mu_{\ell} \mu \cdots \mu_{n}}\left(p ; q_{1}, \cdots q_{l}, k, \cdots q_{n}\right)-I_{\mu_{1} \cdots \mu_{\ell} \mu \cdots \mu_{n}}\left(0 ; q_{1}, \cdots q_{\ell}, 0, \cdots q_{n}\right)\right\} \\
& =I_{\mu_{1} \cdots \mu_{n}}\left(p ; q_{1}, \cdots q_{n}\right)-I_{\mu_{1} \cdots \mu_{n}}\left(p^{\prime} ; q_{1}, \cdots q_{n}\right)+\left.k_{\mu} \partial_{p_{\mu}^{\prime \prime}} I_{\mu_{1} \cdots \mu_{n}}\left(p^{\prime \prime} ; q_{1}, \cdots q_{n}\right)\right|_{p^{\prime \prime}=0},
\end{aligned}
$$

which gives $R^{(\infty)}=0$. Actually, since the integration in (56) is divergent, we should take into account the cutoff functions. We obtain

$$
\begin{aligned}
& R_{\mu_{1} \cdots \mu_{n}}^{\left(\Lambda_{0}\right)}\left(p, p^{\prime}, q_{1} \cdots q_{n}\right)= \\
& \quad I_{\mu_{1} \cdots \mu_{n}}^{\left(0 \Lambda_{0}\right)}\left(p^{\prime} ; q_{1}, \cdots q_{n}\right)\left[K_{0 \Lambda_{0}}\left(p^{\prime}+q_{1}\right)-K_{0 \Lambda_{0}}\left(p+q_{1}\right)\right] \\
& \quad+I_{\mu_{1} \cdots \mu_{n}}^{\left(0 \Lambda_{0}\right)}\left(p ; q_{1}, \cdots q_{n}\right)\left[K_{0 \Lambda_{0}}\left(p^{\prime}-q_{n}\right)-K_{0 \Lambda_{0}}\left(p-q_{n}\right)\right] \\
& +\sum_{\ell=2}^{n-1} I_{\mu_{1} \cdots \mu_{n}}^{\left(0 \Lambda_{0}\right)}\left(p ; q_{1}, \cdots q_{\ell}+k, \cdots q_{n}\right)\left[K_{0 \Lambda_{0}}\left(p^{\prime}+q_{1}+\cdots+q_{\ell-1}\right)-K_{0 \Lambda_{0}}\left(p+q_{1}+\cdots+q_{\ell}\right)\right] \\
& -\left.I_{\mu_{1} \cdots \mu_{n}}\left(0 ; q_{1}, \cdots q_{n}\right) k_{\mu} \partial_{p_{\mu}^{\prime \prime}} K_{0 \Lambda_{0}}\left(p^{\prime \prime}+q_{1}, \cdots p^{\prime \prime}+q_{1}+q_{n-1}\right)\right|_{p^{\prime \prime}=0} .
\end{aligned}
$$

This expression contains differences and derivatives of the cutoff functions. Thus some of the integration momenta $q_{i}$ are of order $Q \sim \Lambda_{0}$. We have to distinguish the two cases in which $G_{\mu_{1} \cdots \mu_{n}}$ corresponds to connected or disconnected graphs.

When $G_{\mu_{1} \cdots \mu_{n}}$ corresponds to connected graphs, the leading contribution to the violation (56) comes from the integration region in which all the intermediate photons $\left(q_{1} \cdots q_{n}\right)$ have large momenta of order $Q$. The resulting asymptotic behaviour of $R^{\left(\Lambda_{0}\right)}$ is given by

$$
R_{\mu_{1} \cdots \mu_{n}}^{\left(\Lambda_{0}\right)}\left(p, p^{\prime}, q_{1} \cdots q_{n}\right) \simeq \frac{k Q}{\Lambda_{0}^{2}} Q^{-n}
$$

This comes from the second order of the expansion of (59) in $p$ and $p^{\prime}$, namely the first derivatives of $I^{\left(0 \Lambda_{0}\right)}$ times the first term of the expansion of the cutoff differences. To evaluate the limit for $\Lambda_{0} \rightarrow \infty$ of $\mathcal{W}^{\mathcal{G}}$ we need the asymptotic behaviour of the connected functions $G_{\mu_{1} \cdots \mu_{n}}\left(q_{1} \cdots q_{n}\right)$ when the momenta become large of order $Q \simeq \Lambda_{0}$. This is provided by the Weinberg theorem which gives

$$
G_{\mu_{1} \cdots \mu_{n}}\left(q_{1} \cdots q_{n}\right) \sim Q^{-2 n} \cdot Q^{4-n},
$$


where the first factor comes from the $n$ photon propagators and the second from the dimensional counting of the vertex with only hard photon lines. The momentum integration in (56) gives a factor $Q^{4 n-4}$, thus the final asymptotic behaviour for $\Lambda_{0} \rightarrow \infty$ is given by

$$
\mathcal{W}^{\mathcal{G}}\left(p, p^{\prime} ; \Lambda_{0}\right) \sim \frac{1}{\Lambda_{0}} \rightarrow 0
$$

We consider now the case in which the function $G_{\mu_{1} \cdots \mu_{n}}$ corresponds to two disconnected graphs, as in Fig. 3b. We exclude at the moment subgraphs contributing to fermion self energy. We have again that the leading contribution is obtained when all $n$ intermediate photon momenta are of order $Q \sim \Lambda_{0}$. In fact the Weinberg theorem gives $G_{\mu_{1} \cdots \mu_{n}} \sim$ $Q^{4} \cdot Q^{4-3 n}$ but the momentum integrations give now $Q^{-4} \cdot Q^{4 n-4}$. Then we obtain the same result in (61). A similar argument holds for the case of any number of disconnected graphs, excluding fermion self energies.

An independent analysis is needed when $G_{\mu_{1} \cdots \mu_{n}}$ contains self energy corrections to the fermion propagators. This is shown for instance in Fig. 3c, in which $m$ photon lines belong to the self energy correction. The leading contribution is obtained when the $m$ photon momenta become large and we have $G_{\mu_{1} \cdots \mu_{n}} \sim Q^{4-3 m}$. From the integration we have $Q^{4-3 m}$. However, since $m<n$, the behaviour of the function $R^{\left(\Lambda_{0}\right)}$ is now given by

$$
R_{\mu_{1} \cdots \mu_{n}}^{\left(\Lambda_{0}\right)}\left(p, p^{\prime}, q_{1} \cdots q_{n}\right) \simeq \frac{k Q}{\Lambda_{0}^{2}} Q^{-m} \cdot Q
$$

The reason for the extra power of $Q$, in comparison to (60), is that not all the momenta $q_{i}$ are large. Thus, when expanding $I^{\left(0 \Lambda_{0}\right)}$ in (59), the derivative with respect to $p$ and $p^{\prime}$ may act on a fermion propagator with soft momentum. These behaviours give a non vanishing contribution to the Ward identity violation $\mathcal{W}^{\mathcal{G}}$. This is expected, since the self energy correction is a relevant contribution and needs to be subtracted. Similarly, the insertions of the photon $k$ in the fermion lines among the $m$ photons of $G_{c}^{\prime}$ give vertex corrections, which are relevant and need subtraction. All these subtractions restore the behaviour in (61), since they force one to take the derivative with respect to $p$ and $p^{\prime}$ of the fermion propagators in the self energy subgraph. In this way one obtains an additional power of $Q^{-1}$. The result is that also in this case $\mathcal{W}^{\mathcal{G}}$ is vanishing as in (61).

In conclusion, one finds that the differences of the cutoff functions in $R^{\left(\Lambda_{0}\right)}$ imply that the Ward identity violation is vanishing as an inverse power of $\Lambda_{0}$. Due to this fact, subtractions of the remaining subdivergences, namely vertex corrections, need not to be analyzed independently since they diverge only logarithmically in $\Lambda_{0}$. This means that we do not need a detailed study of the subdivergences, which relies on the forest technology [15].

The same proof is valid for the general class of Ward identities relating vertices in which any number of photons and fermion pairs are emitted from the blob $G$ in Fig. 3a and Fig. 4.

\section{Photon transversality}

We consider the case in which the photon we want to study is emitted by a fermion loop, as shown in Fig. 5. We denote by $\sigma$ the spinor and Lorentz indices of the lines in $S$. 
The blob corresponds to the sum over all graphs with their subtractions and includes also disconnected graphs. If we are dealing with a relevant vertex (that is $S=\gamma, 3 \gamma, e^{+} e^{-}$), we must take into account the overall subtractions, as required by the boundary conditions. The case with other external photons emitted by the same loop will be discussed later.

Associated with the graph in Fig. 5, there are other contributions coming from the subtractions of subgraphs not contained in the blob. We analyze first those involving the external photon $p$. Consider a cut of the diagrams in the blob which disconnects the $n$ intermediate photons from the external lines $S$. If the lines which are cut are an electronpositron pair or three photons ("relevant intermediate lines"), the subgraph identified by the external photon $p$ and these lines is a relevant vertex, so it must be subtracted. In order to distinguish these cases, we denote by a box $(B)$ the sum of graphs, with their corresponding subtractions, which do not have relevant intermediate lines. From this we have that the graphs in Fig. 5 can be cast in the form of Fig. 6, i.e. the blob can be expanded as a series of boxes. We denote by $\Gamma_{\mu, \sigma}^{\prime b-b o x}(p ; S)$ the generic term of the expansion involving $b$ boxes and by $\Gamma_{\mu, \sigma}^{b-\mathrm{box}}(p ; S)$ the vertex obtained from $\Gamma_{\mu, \sigma}^{\prime b-b o x}(p ; S)$ after having subtracted all the subgraphs involving $p$. We will show that $p_{\mu} \Gamma_{\mu, \sigma}^{b-\text { box }}(p ; S)$ vanishes as a negative power of $\Lambda_{0}$. For this reason, as in the previous case, the subtractions for other subdivergences (e.g. corrections to vertices and propagators in the fermion loop) need not to be considered independently, since they are logarithmic in $\Lambda_{0}$.

In order to prove the transversality we analyze the asymptotic behaviour of the intermediate momentum integrations by using the Weinberg theorem, neglecting all logarithmic corrections. In particular we consider the vertices $B_{n \gamma, m e^{+} e^{-}}\left(q_{1} \cdots ; S\right)$, corresponding to the general box in Fig. 6 in which enter $n$ photons and $m$ fermion pairs with hard momentum. If the state $S$ is "irrelevant", that is $S \neq \gamma, 3 \gamma, e^{+} e^{-}$, from the Weinberg theorem one has the asymptotic behaviour

$$
B_{n \gamma, m e^{+} e^{-}}\left(q_{1} \cdots ; S\right)=\mathcal{O}\left(Q^{-n-3 m}\right),
$$

where all the momenta $q_{i}$ are large of order $Q$ and the momenta in $S$ are finite. If $S$ is "relevant" one has instead

$$
\begin{aligned}
& B_{n \gamma, m e^{+} e^{-}}\left(q_{1} \cdots ; S=\gamma\right)=\mathcal{O}\left(Q^{3-n-3 m}\right) \\
& B_{n \gamma, m e^{+} e^{-}}\left(q_{1} \cdots ; S=3 \gamma\right)=\mathcal{O}\left(Q^{1-n-3 m}\right) \\
& B_{n \gamma, m e^{+} e^{-}}\left(q_{1} \cdots ; S=e^{+} e^{-}\right)=\mathcal{O}\left(Q^{1-n-3 m}\right)
\end{aligned}
$$

From these behaviours we immediately deduce the transversality of $\Gamma_{\mu, \sigma}^{1-b o x}(p ; S)$. For the unsubtracted vertices we have

$$
\begin{aligned}
p_{\mu} \Gamma_{\mu, \sigma}^{\prime \prime-\operatorname{box}}(p ; S)= & \int_{q_{1}} \cdots \int_{q_{n}}(2 \pi)^{4} \delta^{4}\left(\sum_{1}^{n} q_{i}+p\right) \prod_{1}^{n} \frac{K_{0 \Lambda_{0}}\left(q_{i}\right)}{q_{i}^{2}} \\
& \times p_{\mu} \Gamma_{\mu \mu_{1} \cdots \mu_{n}}^{(1)}\left(p, q_{1} \cdots q_{n}\right) B_{\mu_{1} \cdots \mu_{n}}\left(q_{1} \cdots q_{n} ; S\right) .
\end{aligned}
$$

The fermion loop in Fig. 6 gives rise to the one loop vertex $\Gamma_{\mu \mu_{1} \cdots \mu_{n}}^{(1)}$ with $n+1$ photons; for $n=3$ the subtraction is included. From (43) and (44) we have that the longitudinal part of $\Gamma_{\mu, \sigma}^{\prime 1-b o x}(p ; S)$ comes from the integration region of large $q_{i}$, which could compensate the negative power of $\Lambda_{0}$ in $p \cdot \Gamma^{(1)}$. In this case we can use the behaviours in (62) and (63) and obtain the following results. 
a) For the irrelevant vertices the photon is transverse

$$
p_{\mu} \Gamma_{\mu, \sigma}^{1-\operatorname{box}}(p ; S)=\mathcal{O}\left(\frac{1}{\Lambda_{0}}\right) \rightarrow 0
$$

b) For the relevant vertices we find

$$
\begin{aligned}
\frac{p_{\mu} p_{\nu}}{p^{2}} \Gamma_{\mu \nu}^{\prime 1-\operatorname{box}}(p) & =a \Lambda_{0}^{2}+a^{\prime} m^{2}+b p^{2}+\mathcal{O}\left(\frac{p^{4}}{\Lambda_{0}^{2}}\right), \\
p_{\mu} \Gamma_{\mu}^{\prime 1-\operatorname{box}}\left(p, q_{1}, q_{2}\right) & =c \not p+\mathcal{O}\left(p \frac{Q}{\Lambda_{0}}\right), \\
p_{\mu} \Gamma_{\mu \mu_{1} \mu_{2} \mu_{3}}^{\prime 1-\operatorname{box}}\left(p, q_{1}, q_{2}, q_{3}\right) & =d\left(p_{\mu_{1}} \delta_{\mu_{2} \mu_{3}}+\cdots\right)+\mathcal{O}\left(p \frac{Q^{2}}{\Lambda_{0}^{2}}\right),
\end{aligned}
$$

where $Q$ is a combination of the momenta $p$ and $q_{i}$. The constants $a, a^{\prime}, b, c, d$ are relevant couplings which are cancelled after the overall subtractions leaving longitudinal contributions which vanish for $\Lambda_{0} \rightarrow \infty$

$$
\begin{aligned}
\frac{p_{\mu} p_{\nu}}{p^{2}} \Gamma_{\mu \nu}^{1-\operatorname{box}}(p) & =\mathcal{O}\left(\frac{p^{4}}{\Lambda_{0}^{2}}\right) \rightarrow 0, \\
p_{\mu} \Gamma_{\mu}^{1-\operatorname{box}}\left(q_{1}, q_{2}\right) & =\mathcal{O}\left(p \frac{Q}{\Lambda_{0}}\right) \rightarrow 0, \\
p_{\mu} \Gamma_{\mu \mu_{1} \mu_{2} \mu_{3}}^{1-\text { box }}\left(p, q_{1}, q_{2}, q_{3}\right) & =\mathcal{O}\left(p \frac{Q^{2}}{\Lambda_{0}^{2}}\right) \rightarrow 0 .
\end{aligned}
$$

We already noticed that the blob of Fig. 5 is not necessarily connected. In particular we can have some of the $n$ intermediate photons $q_{1} \cdots q_{n}$ disconnected from the external lines $S$. These contributions give for example corrections to the propagators and vertices in the fermion loop. From the Weinberg theorem they have the same behaviours as in (66).

We can now prove by iteration that the behaviour of the diagrams with $b$ boxes is the same as in the case of one box. From Fig. 6 we obtain the contribution for $b+1$ boxes in terms of the one with $b$ boxes as follows

$$
\begin{aligned}
p_{\mu} \Gamma_{\mu, \sigma}^{\prime(b+1)-\operatorname{box}}(p ; S)= & \int_{q_{1}} \int_{q_{2}} K_{0 \Lambda_{0}}\left(q_{1}\right) K_{0 \Lambda_{0}}\left(q_{2}\right)(2 \pi)^{4} \delta^{4}\left(q_{1}+q_{2}+p\right) \\
& \times \operatorname{Tr}\left\{p_{\mu} \Gamma_{\mu}^{b-\mathrm{box}}\left(p ; q_{1}, q_{2}\right) \frac{-i}{q_{1}+m} B_{e^{+} e^{-}}\left(q_{1}, q_{2} ; S\right) \frac{-i}{q_{2}+m}\right\} \\
+ & \int_{q_{1}} \int_{q_{2}} \int_{q_{3}}(2 \pi)^{4} \delta^{4}\left(q_{1}+q_{2}+q_{3}+p\right) \frac{K_{0 \Lambda_{0}}\left(q_{1}\right)}{q_{1}^{2}} \frac{K_{0 \Lambda_{0}}\left(q_{2}\right)}{q_{2}^{2}} \frac{K_{0 \Lambda_{0}}\left(q_{3}\right)}{q_{3}^{2}} \\
& \times p_{\mu} \Gamma_{\mu \mu_{1} \mu_{2} \mu_{3}}^{b-\mathrm{box}}\left(p ; q_{1}, q_{2}, q_{3}\right) B_{\mu_{1} \mu_{2} \mu_{3}}\left(q_{1}, q_{2}, q_{3} ; S\right) .
\end{aligned}
$$

Assuming for the diagrams with $b$ boxes the asymptotic behaviours given in Eq. (66) and using (62)-(63) for the box vertices $B$, we reproduce the assumption for the diagrams with $b+1$ boxes. In the limit $\Lambda_{0} \rightarrow \infty$ we have then proved the transversality of the photon for the graphs with the generic structure of Fig. 5 .

Finally we have to consider the case of graphs in which more than one photon is emitted by the fermion loop. The case with two of such photons $\left(p\right.$ and $\left.p^{\prime}\right)$ is obtained by making 
the substitution $\Gamma_{\mu \mu_{1} \cdots \mu_{n}}^{(1)}\left(p, q_{1} \cdots q_{n}\right) \rightarrow \Gamma_{\mu \nu \mu_{1} \cdots \mu_{n}}^{(1)}\left(p, p^{\prime}, q_{1} \cdots q_{n}\right)$. in (64). In this case the relevant intermediate lines are given only by two photons and the proof proceeds as before. For more than two external photons emitted by the first fermion loop we don't have intermediate relevant lines and the proof reduces to the analysis of the one box contribution and can be done as in the previous case.

\section{Perturbative renormalization}

Perturbative renormalization is essentially based on power counting. In four space time dimensions a given vertex $\Gamma_{c_{1} \cdots c_{n}}$ with $n_{A}$ photons and $n_{\psi}$ pairs of fermions has dimension in mass given by

$$
\operatorname{dim} \Gamma_{c_{1} \cdots c_{n}}=4-n_{A}-3 n_{\psi} .
$$

To prove perturbative renormalizability we shall follow the method of Ref. [10]. We analyse the UV finiteness of the loop expansion contributions which are obtained by iterating the integral equations corresponding to the evolution equations (6) and the boundary conditions (13) and (14).

\section{Integral equations}

For the relevant couplings we have the integral equations

$$
\begin{aligned}
\sigma_{i}(\Lambda) & =e \delta_{i, e}+\int_{0}^{\Lambda} \frac{d \lambda}{\lambda} R_{i}(\lambda) \\
R_{i}(\lambda) & =-\frac{1}{2} \int_{q} M_{b a}(q ; \lambda) \bar{\Gamma}_{a b, i}(-q, q ; \lambda),
\end{aligned}
$$

where $\sigma_{e}(0)=e$ is the only coupling different from zero. The auxiliary vertices $\bar{\Gamma}_{a b, i}$ are given in Appendix B.

For the irrelevant vertices we have two cases according to whether $4-n_{A}-3 n_{\psi}<0$ or $4-n_{A}-3 n_{\psi} \geq 0$. In the first case the vertices satisfy the integral equation

$$
\Gamma_{c_{1} \cdots c_{n}}\left(p_{1}, \cdots p_{n} ; \Lambda\right)=-\int_{\Lambda}^{\Lambda_{0}} \frac{d \lambda}{\lambda} I_{c_{1} \cdots c_{n}}\left(p_{1}, \cdots p_{n} ; \lambda\right)
$$

where

$$
I_{c_{1} \cdots c_{n}}\left(p_{1}, \cdots p_{n} ; \lambda\right)=-\frac{1}{2} \int_{q} M_{b a}(q ; \lambda) \bar{\Gamma}_{a b, c_{1} \cdots c_{n}}\left(-q, q ; p_{1}, \cdots p_{n} ; \lambda\right) .
$$

In the second case we have the five vertices $\Sigma_{i}$ given by

$$
\Sigma_{L}(p ; \Lambda), \quad \Sigma_{T}(p ; \Lambda), \quad \Sigma_{\psi}(p ; \Lambda), \quad \Sigma_{\mu}\left(p, p^{\prime} ; \Lambda\right), \quad \Sigma_{\mu_{1} \cdots \mu_{4}}\left(p_{1}, \cdots p_{4} ; \Lambda\right),
$$

which satisfy the integral equations

$$
\Sigma_{i}(p \cdots ; \Lambda)=-\int_{\Lambda}^{\Lambda_{0}} \frac{d \lambda}{\lambda} I_{i}^{s}(p \cdots ; \lambda),
$$


where the subtracted integrands $I_{i}^{s}$ are given in Appendix B. For instance for $\Sigma_{L}(p ; \Lambda)$ we have

$$
\begin{aligned}
& I_{L}^{s}(p ; \lambda)=I_{L}(p ; \lambda)-I_{L}(0 ; \lambda)-\left.p^{2} \partial_{p^{\prime 2}} I_{L}\left(p^{\prime} ; \lambda\right)\right|_{p^{\prime}=\bar{p}} \\
& =\left(p \partial_{p}\right)^{4} \int_{0}^{1} \frac{d x(1-x)^{3}}{3 ! x^{4}} I_{L}(x p ; \lambda)-\frac{1}{2}\left(p \partial_{p^{\prime}}\right)^{2}\left[\left.I_{L}\left(p^{\prime} ; \lambda\right)\right|_{p^{\prime}=\bar{p}}-\left.I_{L}\left(p^{\prime} ; \lambda\right)\right|_{p^{\prime}=0}\right] \\
& +2(p \bar{p})^{2}\left(\partial_{\bar{p}^{2}}\right)^{2} I_{L}(\bar{p} ; \lambda)
\end{aligned}
$$

where

$$
I_{L}(p ; \lambda)=-\frac{1}{2} \int_{q} M_{b a}(q ; \lambda) \bar{\Gamma}_{a b, L}(-q, q ;-p, p ; \lambda) .
$$

As we see from this expression, since the subtracted vertex vanishes with its derivative, we can factorize four powers of momentum and the remaining function has negative dimension.

\section{Perturbative analysis}

To prove that the theory is renormalizable we must show that the integral equations give a finite result in the limit $\Lambda_{0} \rightarrow \infty$. This can be done perturbatively by iterating Eq. (68a), (69) and (70), in which we set $\Lambda_{0} \rightarrow \infty$. In order to see that the integrations over $\lambda$ are convergent, we have to estimate the behaviour of the integrands for large $\lambda$. The analysis can be simplified, following Polchinski, by introducing the norm

$$
|f|_{\lambda} \equiv \operatorname{Max}_{p_{i}^{2}<c \lambda^{2}}\left|f\left(p_{1}, \cdots p_{n} ; \lambda\right)\right|
$$

which allows us to ignore the momentum dependence. Since the $\Lambda$-dependence is fixed only by the number of photons and fermions, to simplify the notation, we indicate in the vertices only the numbers $n_{A}$ and $n_{\psi}$

$$
\left|\Gamma_{c_{1} \cdots c_{n}}\left(p_{1} \cdots p_{4} ; \Lambda\right)\right|_{\Lambda} \equiv\left|\Gamma\left(n_{A}, n_{\psi}\right)\right|_{\Lambda} .
$$

We shall deduce perturbative renormalization by proving by induction on the number of loops that this norm satisfies power counting. Namely, at loop $\ell$, we assume for large $\Lambda$ the following behaviours

$$
\begin{aligned}
& \sigma_{m_{A}}^{(\ell)}(\Lambda)=\mathcal{O}\left(*^{\epsilon}\right), \quad \sigma_{\mathbb{1}_{\psi}}^{(\ell)}(*)=\mathcal{O}(*), \\
& \sigma_{A}^{(\ell)}(\Lambda), \quad \sigma_{\psi}^{(\ell)}(\Lambda), \quad \sigma_{\alpha}^{(\ell)}(\Lambda), \quad \sigma_{e}^{(\ell)}(\Lambda), \quad \sigma_{4}^{(\ell)}(\Lambda)=\mathcal{O}(\infty), \\
& \left|\Gamma^{(\ell)}\left(n_{A}, n_{\psi}\right)\right|_{\Lambda}=\mathcal{O}\left(*^{\triangle-} \backslash_{\mathcal{A}}-\ni \backslash_{\psi}\right), \quad \triangle-\backslash_{\mathcal{A}}-\ni \backslash_{\psi}<\prime, \\
& \left|\Sigma_{T, L}^{(\ell)}\right|_{\Lambda}=\mathcal{O}\left(*^{\epsilon}\right), \quad\left| \pm_{\psi}^{(\ell)}\right|_{*}=\mathcal{O}(*), \\
& \left|\Sigma_{\mu}^{(\ell)}\right|_{\Lambda}=\mathcal{O}(\infty), \quad\left| \pm_{\mu_{\infty} \cdots \mu_{\Delta}}^{(\ell)}\right|_{*}=\mathcal{O}(\infty) .
\end{aligned}
$$

We neglect for simplicity all possible $\ell$-dependent powers of $\log \frac{\Lambda}{m}$. These behaviours are satisfied for the one loop case discussed in section 3 . We now proceed by iteration and prove that the behaviours $(\sqrt{73})$ are reproduced at the loop $\ell+1$. To do this we need to give a bound of the r.h.s. of the integral equation by estimating $R_{i}(\lambda)$ in (68a and the norms of $I_{c_{1} \cdots c_{n}}\left(p_{1}, \cdots p_{n} ; \lambda\right)$ and $I_{i}^{s}(p, \cdots ; \lambda)$ in $(69)$ and $(70)$. 
First of all we notice that the norm of the auxiliary vertices have the same behaviours as the corresponding vertices, as can be seen from their definition. Thus we have

$$
\begin{aligned}
& \left|\bar{\Gamma}_{\alpha \beta, c_{1} \cdots c_{n}}^{(\ell)}\left(-q, q ; p_{1}, \cdots p_{n} ; \lambda\right)\right|_{\lambda}=\mathcal{O}\left(\lambda^{\triangle-\backslash_{\mathcal{A}}-\ni \backslash_{\psi}-\ni}\right), \\
& \left|\bar{\Gamma}_{\mu \nu, c_{1} \cdots c_{n}}^{(\ell)}\left(-q, q ; p_{1}, \cdots p_{n} ; \lambda\right)\right|_{\lambda}=\mathcal{O}\left(\lambda^{\triangle-\backslash_{\mathcal{A}}-\ni \backslash_{\psi}-\epsilon}\right) .
\end{aligned}
$$

Then we observe that the irrelevant vertices with positive dimensions are given by subtracted auxiliary vertices. These are obtained by taking derivatives of the auxiliary vertices with respect to the external momenta. This reduces the powers of $\lambda$

$$
\left|\partial_{p_{i}}^{m} \Gamma_{c_{1} \cdots c_{n}}^{(\ell)}\left(p_{1}, \cdots p_{n} ; \lambda\right)\right|_{\lambda}=\mathcal{O}\left(\lambda^{\triangle-\backslash_{\mathcal{A}}-\ni \backslash_{\psi}-\Uparrow \mathbb{}}\right) .
$$

The proof of this is given for instance in Ref. [10]. Notice that from the assumptions we find

$$
\left|\Pi^{(\ell)}(q ; \lambda) \frac{1}{\not q+m}\right|_{\lambda}=\mathcal{O}(\infty),\left.\left.\quad\right|_{\mathcal{T}, \mathcal{L}} ^{(\ell)}(\amalg ; \lambda) \frac{\infty}{\amalg^{\epsilon}}\right|_{\lambda}=\mathcal{O}(\infty),
$$

thus, using (24), $M_{a b}$ gives a factor $\lambda^{-1}$ for fermions and $\lambda^{-2}$ for photons. We now obtain from the iterative solution of the integral equations the norm of vertices and couplings at loop $\ell+1$.

We discuss first the relevant couplings. Consider for instance the iterative equation for $\sigma_{m_{A}}(\Lambda)$. We have

$$
R_{m_{A}}^{(\ell)}(\lambda)=-\frac{1}{2} \int_{q} M_{b a}^{\left(\ell^{\prime}\right)}(q ; \lambda) \bar{\Gamma}_{a b L}^{\left(\ell-\ell^{\prime}\right)}(-q, q ; 0,0 ; \lambda)
$$

which gives

$$
R_{m_{A}}^{(\ell)}(\lambda) \lesssim \lambda^{2}\left|\Gamma^{(\ell)}(4,0)\right|_{\lambda}+\lambda^{3}\left|\Gamma^{(\ell)}(2,1)\right|_{\lambda}=\mathcal{O}\left(\lambda^{\in}\right),
$$

where we neglected the self energy contributions due to (76) and we have used (73) for the vertex $\left|\Gamma^{(\ell)}\left(n_{A}, n_{\psi}\right)\right|_{\lambda}$. We find then

$$
\sigma_{m_{A}}^{(\ell+1)}(\Lambda) \lesssim \int_{0}^{\Lambda} \frac{d \lambda}{\lambda}\left(\lambda^{2}\left|\Gamma^{(\ell)}(4,0)\right|_{\lambda}+\lambda^{3}\left|\Gamma^{(\ell)}(2,1)\right|_{\lambda}\right)=\mathcal{O}\left(*^{\epsilon}\right) .
$$

Similar results are obtained for the other couplings. Notice that in this case the integrand grows with $\lambda$ and the result is dominated by the upper limit $\Lambda$.

We consider now the case of irrelevant vertices. In this case the $\lambda$-integration goes up to infinity (for $\Lambda_{0} \rightarrow \infty$ ). We treat separately the irrelevant vertices with negative and positive dimensions. For the first ones we have

$$
\begin{aligned}
& \left|I^{(\ell)}\left(n_{A}, n_{\psi}\right)\right|_{\lambda} \simeq \lambda^{2}\left|\Gamma^{(\ell)}\left(n_{A}+2, n_{\psi}\right)\right|_{\lambda}+\lambda^{3}\left|\Gamma^{(\ell)}\left(n_{A}, n_{\psi}+1\right)\right|_{\lambda}=\mathcal{O}\left(\lambda^{\triangle-\backslash_{\mathcal{A}}-\ni \backslash_{\psi}}\right), \\
& \left|\Gamma^{(\ell+1)}\left(n_{A}, n_{\psi}\right)\right|_{\Lambda} \lesssim \int_{\Lambda}^{\infty} \frac{d \lambda}{\lambda}\left|I^{(\ell)}\left(n_{A}, n_{\psi}\right)\right|_{\lambda}=\mathcal{O}\left(*^{\triangle-} \backslash_{\mathcal{A}}-\ni \backslash_{\psi}\right) .
\end{aligned}
$$

Again self energy contributions can be ignored. Since $4-n_{A}-3 n_{\psi}<0$ the integration over $\lambda$ is convergent and the integral is dominated by the lower limit, thus reproducing at loop $\ell+1$ the Ansatz (73).

We now study the irrelevant vertices with non negative dimension. Consider for instance the case of $\Sigma_{L}^{(\ell+1)}(p ; \Lambda)$ (see $(\sqrt[72]{ })$ and $\left.(\sqrt{71})\right)$. Due to the subtractions the integrand can be 
expressed as fourth derivative with respect to the external momenta for which we can use (75). We have then

$$
\left|I_{L}^{s(\ell)}(p ; \lambda)\right|_{\lambda} \sim p^{4}\left|\partial^{4} I_{L}^{(\ell)}(p ; \lambda)\right|_{\lambda}=p^{4} \mathcal{O}\left(\lambda^{-\epsilon}\right)
$$

for $p^{2}<\lambda^{2}$. We find

$$
\left|\Sigma_{L}^{(\ell+1)}(p ; \Lambda)\right|_{\Lambda} \leq \Lambda^{4} \int_{\Lambda}^{\infty} \frac{d \lambda}{\lambda}\left|\partial^{4} I_{L}^{(\ell)}(p ; \lambda)\right|_{\lambda}=\mathcal{O}\left(*^{\epsilon}\right)
$$

We see here that the subtractions make the $\lambda$-integration convergent and dominated by the lower limit $\Lambda$. The positive power of $\Lambda$ in the Ansatz (73) is recovered from $\Lambda^{4}$ coming by maximizing the factor $p^{4}$ in (78).

\section{Conclusions}

In this paper we have analyzed how the Ward identities emerge in the Wilson renormalization group formulation of QED. The main feature is that this gauge symmetry must be implemented at the level of the relevant part of the physical effective action. This implies that the seven relevant couplings $\sigma_{i}(\Lambda)$ must be fixed at $\Lambda=0$, according to (1) or (13). We have shown that in perturbation theory the renormalization group equations ensure that the full effective action at $\Lambda=0$ and $\Lambda_{0} \rightarrow \infty$ satisfies the Ward identities. This fact is deeply connected to the renormalizability of the theory, i.e. to the large momentum behaviour of the integrands of Feynman diagrams. The UV cutoff $\Lambda_{0}$ must be kept when we study graphs which do not include the subtractions imposed by the boundary conditions in (1). We find that the Ward identity violating contributions involve differences of the cutoff functions at $\Lambda=0$ which force the integration momenta around the UV cutoff. Thus their behaviours for large $\Lambda_{0}$ are obtained by estimating the large momentum limit of Feynman diagram integrands, which are given by the Weinberg theorem. For the vertex identities, the result is that for $\Lambda_{0} \rightarrow \infty$ the violation, $\mathcal{W}^{\mathcal{G}}\left(p, p^{\prime} ; \Lambda_{0}\right)$, is vanishing as an inverse power of the cutoff. This is the reason why we can avoid a detailed study of other subtractions, which behave logarithmically in $\Lambda_{0}$, and the relative forest expansion. For the photon transversality, we have that for $\Lambda_{0} \rightarrow \infty$ the possible non vanishing longitudinal contributions are given by relevant contributions, i.e. by polynomial at most of quadratic degree in the external momenta, which are automatically cancelled by subtractions.

We want to emphasize that, in order to satisfy the Ward identities, one must require not only $\Lambda_{0} \rightarrow \infty$ but also $\Lambda=0$. For $\Lambda \neq 0$ the difference of cutoff functions does not force the integration momentum in the UV region only and the violation of the Ward identities would not be polynomial in the external momenta. One can verify at the one loop level that even for $\Lambda_{0} \rightarrow \infty$ the Ward identities are not satisfied as long as $\Lambda \neq 0$. See for instance Eq. (32) for the vertex identity.

In conclusion the functional $\Gamma[A, \psi, \bar{\psi} ; \Lambda]$ does not satisfy Ward identities except at the physical point $\Lambda=0$ and $\Lambda_{0} \rightarrow \infty$. At the UV scale $\Lambda=\Lambda_{0}$ this functional is given only

by the relevant part, i.e. by the seven couplings $\sigma_{i}\left(\Lambda_{0}\right)$ which are the couplings $\sigma_{i}^{B}$ in the effective Lagrangian at the UV scale in Eq. (3). Although these couplings do not satisfy 
Ward identities, they are functionally related since they lead to the physical effective action which fulfils these identities. In Eqs. (29),(31),(35) and (40) we have computed the seven couplings $\sigma_{i}(\Lambda)$ at one loop for large $\Lambda$, using a sharp momentum cutoff.

In the last part of the paper we have given a simple proof of perturbative renormalizability of QED in this framework. We used the same method of Ref. [10]. The proof is based on the fact that Feynman graphs obtained by iteratively solving the evolution equations are organized in such a way that the loop momenta are ordered. It is then possible to analyze their ultraviolet behaviour by iterative methods. The necessary subtractions and the corresponding counterterms are automatically generated in the process of fixing the physical conditions for the "relevant" vertices. The proof of perturbative renormalizability is simply based on dimensional arguments and does not require the usual analysis of topological properties of Feynman graphs.

\section{Acknowledgements}

We have benefited greatly from discussions with C. Becchi, G. Bottazzi and M. Tonin. 


\section{Appendix A}

We derive here the evolution equation for the cutoff effective action. Taking into account that

$$
\Lambda \partial_{\Lambda} \Gamma[\Phi ; \Lambda]=-\Lambda \partial_{\Lambda} W[J ; \Lambda]+\Lambda \partial_{\Lambda} W[0 ; \Lambda]
$$

the evolution equation for the cutoff effective action is given by

$$
\begin{aligned}
\Lambda \partial_{\Lambda}\{ & \left.\Gamma[\Phi ; \Lambda]-\frac{1}{2} \int_{p} \Phi_{a}(-p) D_{a b}^{-1}(p ; \Lambda) \Phi_{b}(p)\right\} \\
& =\Lambda \partial_{\Lambda} W[0 ; \Lambda]+\frac{1}{2}(2 \pi)^{8} \int_{q} \Lambda \partial_{\Lambda} D_{b a}^{-1}(q ; \Lambda) \frac{\delta^{2} W[J ; \Lambda]}{\delta J_{b}(q) \delta J_{a}(-q)} .
\end{aligned}
$$

The functional in the integrand is the inverse of $\delta^{2} \Gamma[\Phi ; \Lambda] / \delta \Phi_{a}(q) \delta \Phi_{b}\left(q^{\prime}\right)$

$$
\delta^{4}\left(q+q^{\prime}\right) \delta_{a b}=(2 \pi)^{8} \int_{q^{\prime \prime}} \frac{\delta^{2} W[J]}{\delta J_{b}\left(q^{\prime}\right) \delta J_{c}\left(-q^{\prime \prime}\right)} \frac{\delta^{2} \Gamma[\Phi]}{\delta \Phi_{c}\left(q^{\prime \prime}\right) \delta \Phi_{a}(q)}(-)^{\delta_{a}}
$$

where $\delta_{a}$ is one if $a$ is a fermion index and zero otherwise. We isolate, similarly to Eq. (5), the two point function contribution in the functional $W[J ; \Lambda]$

$$
(2 \pi)^{8} \frac{\delta^{2} W[J ; \Lambda]}{\delta J_{a}(q) \delta J_{b}\left(q^{\prime}\right)}=(2 \pi)^{4} \delta^{4}\left(q+q^{\prime}\right)(-)^{\delta_{a}} \Delta_{b a}(q ; \Lambda)+W_{b a}^{i n t}\left[q^{\prime}, q ; J\right] .
$$

Notice that the two point function contribution cancels the constant term $\Lambda \partial W[0 ; \Lambda] / \partial \Lambda$ in (79). Then, from (80) we express $W_{a b}^{i n t}$ as functional of $\Phi(p)$

$$
W_{a b}^{i n t}\left[-q, q^{\prime} ; J\right]=-\Delta_{a c}(q ; \Lambda) \bar{\Gamma}_{c d}\left[-q, q^{\prime} ; \Phi\right] \Delta_{d b}\left(q^{\prime} ; \Lambda\right),
$$

where the auxiliary functional $\bar{\Gamma}$ satisfies the Eq. (8) (see Fig. 1). In conclusion we obtain Eq. (6). 


\section{Appendix B}

The auxiliary vertices $\bar{\Gamma}_{a b, i}$ for the equation (68b) are given by

$$
\begin{aligned}
& \bar{\Gamma}_{a b, m_{\psi}}(-q, q ; \lambda)=-i \frac{1}{4} \delta_{\alpha \beta} \bar{\Gamma}_{a b, \alpha \beta}(-q, q ;-\bar{p}, \bar{p} ; \lambda) \quad \not p=-m, \\
& \bar{\Gamma}_{a b, \psi}(-q, q ; \lambda)=\left.i \frac{1}{16}\left(\gamma_{\mu}\right)_{\beta \alpha} \frac{\partial}{\partial p_{\mu}^{\prime}} \bar{\Gamma}_{a b, \alpha \beta}\left(-q, q ;-p^{\prime}, p^{\prime} ; \lambda\right)\right|_{p^{\prime}=0}, \\
& \bar{\Gamma}_{a b, e}(-q, q ; \lambda)=i \frac{1}{16}\left(\gamma_{\mu}\right)_{\beta \alpha} \bar{\Gamma}_{a b, \alpha \beta \mu}(-q, q ; 0,0,0 ; \lambda), \\
& \bar{\Gamma}_{a b, m_{A}}(-q, q ; \lambda)=\bar{\Gamma}_{a b, L}(-q, q ; 0,0 ; \lambda), \\
& \bar{\Gamma}_{a b, \alpha}(-q, q ; \lambda)=\left.\frac{\partial}{\partial p^{2}} \bar{\Gamma}_{a b, L}(-q, q ;-p, p ; \lambda)\right|_{p=\bar{p}} \quad \bar{p}^{2}=\mu^{2}, \\
& \bar{\Gamma}_{a b, A}(-q, q ; \lambda)=\left.\frac{\partial}{\partial p^{2}} \bar{\Gamma}_{a b, T}(-q, q ;-p, p ; \lambda)\right|_{p=\bar{p}} \quad \bar{p}^{2}=\mu^{2} \\
& \bar{\Gamma}_{a b, 4}(-q, q ; \lambda)=\frac{1}{24} \bar{\Gamma}_{a b, \mu \mu \nu \nu}(-q, q ; 0,0,0,0 ; \lambda),
\end{aligned}
$$

where we introduced the notations

$$
\bar{\Gamma}_{a b, L}=\frac{p_{\mu} p_{\nu}}{p^{2}} \bar{\Gamma}_{a b, \mu \nu}, \quad \bar{\Gamma}_{a b, T}=\frac{1}{3}\left(\delta_{\mu \nu}-4 \frac{p_{\mu} p_{\nu}}{p^{2}}\right) \bar{\Gamma}_{a b, \mu \nu} .
$$

The integrands $I_{i}^{s}$ for the five vertices $\Sigma_{i}$ (see (70)) are given by

$$
I_{i}^{s}\left(p_{1} \cdots p_{n} ; \lambda\right)=-\frac{1}{2} \int_{q} M_{b a}(q ; \lambda) \bar{\Gamma}_{a b, i}^{s}\left(-q, q ; p_{1} \cdots p_{n} ; \lambda\right),
$$

where the subtracted auxiliary vertices are

$$
\begin{aligned}
& \bar{\Gamma}_{a b, \psi}^{s}=\bar{\Gamma}_{a b, \alpha \beta}(-q, q ;-p, p ; \lambda)-\bar{\Gamma}_{a b, \alpha \beta}(-q, q ;-\bar{p}, \bar{p} ; \lambda) \\
&-\left.\left(p_{\mu}-\frac{m}{4} \gamma_{\mu}\right)_{\alpha \alpha^{\prime}} \frac{\partial}{\partial p^{\prime}} \bar{\Gamma}_{a b, \alpha^{\prime} \beta}\left(-q, q ;-p^{\prime}, p^{\prime} ; \lambda\right)\right|_{p^{\prime}=0} \sim(p \partial)^{2} \bar{\Gamma} \quad \not p=-m, \\
& \bar{\Gamma}_{a b, \mu}^{s}=\bar{\Gamma}_{a b, \alpha \beta \mu}\left(-q, q ;-p, p^{\prime}, p-p^{\prime} ; \lambda\right)-\bar{\Gamma}_{a b, \alpha \beta \mu}(-q, q ; 0,0,0 ; \lambda) \sim p \partial \bar{\Gamma}, \\
& \bar{\Gamma}_{a b, L}^{s}=\bar{\Gamma}_{a b, L}(-q, q ;-p, p ; \lambda)-\bar{\Gamma}_{a b, L}(-q, q ; 0,0 ; \lambda) \\
&-\left.p^{2} \frac{\partial}{\partial p^{\prime 2}} \bar{\Gamma}_{a b, L}\left(-q, q ;-p^{\prime}, p^{\prime} ; \lambda\right)\right|_{p^{\prime 2}=\mu^{2}} \sim(p \partial)^{4} \bar{\Gamma}, \\
& \bar{\Gamma}_{a b, T}^{s}=\bar{\Gamma}_{a b, T}(-q, q ;-p, p ; \lambda)-\left.p^{2} \frac{\partial}{\partial p^{\prime 2}} \bar{\Gamma}_{a b, T}\left(-q, q ;-p^{\prime}, p^{\prime} ; \lambda\right)\right|_{p^{\prime 2}=\mu^{2}} \sim(p \partial)^{4} \bar{\Gamma}, \\
& \bar{\Gamma}_{a b, \mu_{1} \cdots \mu_{4}}^{s}=\bar{\Gamma}_{a b, \mu_{1} \cdots \mu_{4}}\left(-q, q ; p_{1} \cdots p_{4} ; \lambda\right)-\bar{\Gamma}_{a b, \mu_{1} \cdots \mu_{4}}(-q, q ; 0 \cdots 0 ; \lambda) \sim(p \partial)^{2} \bar{\Gamma} .
\end{aligned}
$$




\section{References}

[1] K. Wilson, Phys. Rev. D 14 (1974) 2455; K. Wilson, in "New Phenomena in Subnuclear Physics", ed. A. Zichichi, Plenum, New York 1977.

[2] G. Costa, T. Marinucci, J. Julve and M. Tonin, Il Nuovo Cimento 38 A (1977) 373; S. Aoyama and M. Tonin, Nucl. Phys. B179 (1981) 293; G. Bandelloni, C. Becchi, A. Blasi and R. Collina, Comm. Math. Phys. 72 (1980) 239; J.L. Trueman, Phys. Lett. 88B (1979) 331; S. Gottlieb and J.T. Donohue, Phys. Rev. D 20 (1979) 3378; G. Bonneau, Nucl. Phys. B177 (1981) 523.

[3] L. Maiani, Nucl. Phys. B (Proc. Suppl.) 29B,C (1992) 33; J. Smit, Nucl. Phys. B (Proc. Suppl.) 29B,C (1992) 83; I. Montvay, Nucl. Phys. B (Proc. Suppl.) 29B,C (1992) 159; M.F.L. Golterman, D.N. Petcher and E. Rivas, Nucl. Phys. B (Proc. Suppl.) 29B,C (1992) 193, and refs. therein.

[4] K.G. Wilson, Phys. Rev. B 4 (1971) 3174,3184; K.G. Wilson and J.G. Kogut, Phys. Rep. 12 (1974) 75.

[5] J. Polchinski, Nucl. Phys. B231 (1984) 269.

[6] G. Gallavotti, Rev. Mod. Phys. 57 (1985) 471.

[7] C. Becchi, Lectures on the renormalization of gauge theories, in: Relativity, groups and topology II (Les Houches 1983) Eds. B.S. DeWitt and R. Stora (Elsevier Science Pub. 1984); C. Becchi, On the construction of renormalized quantum field theory using renormalization group techniques, Lectures notes of the 1991 Italian doctoral school in Parma, Genova preprint.

[8] B.J. Warr, Ann. Phys. (NY) 183 (1988) 1,89; G. Keller and C. Kopper, Phys. Lett. 273B (1991) 323.

[9] G. Keller, C. Kopper and M. Salmhofer, Helv. Phys. Acta 65 (1992) 32.

[10] M. Bonini, M. D’Attanasio and G. Marchesini, Perturbative renormalization and infrared finiteness in the Wilson renormalization group: the massless scalar case, Parma preprint, UPRF 92-360, Nucl. Phys. B to be published.

[11] C. Wetterich, Phys. Lett. 301B (1993) 90.

[12] L. Girardello and A. Zaffaroni, Exact renormalization group equation and decoupling in quantum field theory, Milano-SISSA/EP preprint 1993.

[13] J. Feldman, T. Hurd, L. Rosen and J. Wright, QED: A proof of renormalizability, Lecture Notes in Physics 312, Springer 1988; T. Hurd, Comm. Math. Phys. 125 (1989) 515.

[14] S. Weinberg, Phys. Rev. D 118 (1960) 838.

[15] W. Zimmermann, Comm. Math. Phys. 15 (1969) 208; W. Zimmermann, in Lectures on elementary particles and quantum field theory, Proc. 1970 Brandeis Summer Institute (ed. S. Deser et al.), MIT Press, Cambridge, Massachusetts. 


\section{Figure Captions}

Figure 1: Graphical representation of Eq. (8) defining the auxiliary functional $\bar{\Gamma}_{a b}\left[q, q^{\prime} ; \Phi\right]$. Solid lines represent in this case both fermions and photons. The dashed blob represents the functional $\bar{\Gamma}_{a b}^{i n t}\left[q, q^{\prime} ; \Phi\right]$ defined in Eq. (5).

Figure 2: a) Graphical representation of the auxiliary vertex at zero loop for the electron emitting $n$ photons.

b) Auxiliary vertex giving the electron propagator at one loop (see Eq.(27)).

Figure 3: a) General graph for the fermion propagator. The function $G$ corresponds to the sum of connected and disconnected graphs.

b) Example in which the function $G$ is disconnected. No fermion self energy subgraphs are here included.

c) Example in which the function $G$ is disconnected. In this case $G_{c}^{\prime}$ gives subgraphs of fermion self energy corrections.

Figure 4: General graph for the fermion-photon vertex.

Figure 5: General graph for a photon $p$ emitted by a fermion loop. The other external lines in $S$ are not emitted by this loop.

Figure 6: Expansion for the graph of Fig. 5. The box is defined as the sum of diagrams for which a vertical line cuts intermediate propagators which are not "relevant lines", i.e. a single fermion pair or three photons only. 